\title{
A Multi-Time Scale Approach to Remaining Useful Life Prediction in Rolling Bearing
}

\author{
Yuning Qian ${ }^{1,2}$, Ruqiang Yan $^{1,3, *}$, and Robert X. Gao ${ }^{3}$ \\ ${ }^{1}$ School of Instrument Science and Engineering \\ Southeast University \\ Nanjing, Jiangsu 210096, P.R. China \\ Email: inter101010@sina.com; ruqiang@seu.edu.cn \\ Phone: +86 (135) 84054760 \\ ${ }^{2}$ Nanjing Research Institute of Electronics Technology \\ Nanjing 210039, P.R. China \\ ${ }^{3}$ Department of Mechanical and Aerospace Engineering \\ Case Western Reserve University \\ Cleveland, OH 44106, USA \\ Email: Robert.gao@,case.edu \\ * Corresponding author
}

Abstract-This paper presents a novel multi-time scale approach to bearing defect tracking and remaining useful life (RUL) prediction, which integrates enhanced phase space warping (PSW) with a modified Paris crack growth model. As a data-driven method, PSW describes the dynamical behavior of the bearing being tested on a fast-time scale, whereas the Paris crack growth model, as a physics-based model, characterizes the bearing's defect propagation on a slow-time scale. Theoretically, PSW constructs a tracking metric by evaluating the phase space trajectory warping of the bearing vibration data, and establishes a correlation between measurement on a fast-time scale and defect growth variables on a slow-time scale.

Furthermore, PSW is enhanced by a multi-dimensional auto-regression (AR) model for improved accuracy in defect tracking. Also, the Paris crack growth model is modified by a time-piecewise algorithm for real-time RUL prediction. Case studies performed on two run-to-failure experiments indicate that the developed technique is effective in tracking the 
evolution of bearing defects and accurately predict the bearing RUL, thus contributing to the literature of bearing prognosis .

Keywords-Multi-time scale modeling; Enhanced phase space warping; Modified Paris crack growth model; RUL prediction; Rolling bearing

\section{Introduction}

Over the past decades, machine maintenance strategies have progressed from a condition-based maintenance (CBM) strategy towards a futuristic view of intelligent, predictive maintenance [1]. This requires real-time failure tracking and reliable remaining useful life (RUL) prediction before failure occurrence. Particularly, since bearings are critical element to almost all forms of rotating machines, bearing health monitoring has a significant effect on the working status of the machine system. Accordingly, bearing failure tracking and RUL prediction have received considerable attention in recent years.

The majority of prior research reported in the literature on bearing failure tracking and RUL prediction took a data-driven approach, with models derived from data collected by sensors on running machines to monitor bearing degradation and predict its RUL. Several time-frequency methods, such as wavelet transform [2, 3] and empirical mode decomposition (EMD) [4], have been reported to extract features from vibration signals for bearing degradation tracking. In addition, support vector data description [3], local and nonlocal preserving projection [5], Gaussian mixture model [6], hidden Markov model [7] and Kolmogorov-Smirnov test [8] have been investigated to construct health indicators for describing the bearing degradation progress. Based on the obtained degradation features, 
various data-driven prediction models were applied to estimating the RUL of bearings. For example, neural networks [2, 9], Kalman filter [10, 11], Bayesian algorithms [12], particle filter [13-16], support vector regression [17] and hidden Markov model [18] were all investigated for bearing RUL estimation and other prediction-related applications. The main advantage of these data-driven techniques is that they reflect realistically the dynamical behavior of the system being monitored, and lend themselves readily to adjustment of model parameters to capture the trend of the sensor signals. A common limitation associated with these techniques is that they do not establish a theoretical or functional link between the physical damage state and the feature vectors obtained from the models, due to the "data only" nature. In comparison, physics-based prediction models, such as Paris crack growth model [19, 20], Forman crack growth model [21, 22] and spall progression model [23], describe variations in the physical states and evolution of structural damage according to the mechanistic law that govern the material response of a bearing to loading conditions that it is subject to. The constraint in developing physics-based prediction models is that they require explicit analytical understanding of the damage evolution, which is obtained from historical data gathered by sensors beforehand. Once a physics-based model is established, relevant model parameters cannot be adjusted in real time. This means that the actual change in states as reflected in the measured signals will not be utilized in real time. From a "time-scale" perspective, data-driven methods extract features from real-time sensor data, thus is considered as reflecting the characteristics of a dynamical system on a fast time-scale. In comparison, physics-based methods describe the physical state evolution on a slow time-scale. A dynamical system is modeled as a hierarchical system consisting of both a fast and a slow 
time subsystem [24]. Using either data-driven approach or physics-based approach alone can only address partial or a specific aspect of the entire system. This observation motivates the study of an integrated method for bearing RUL prediction, which combines the strength of both data-driven and physics-based techniques $[25,26]$. In this paper, phase space warping (PSW) $[24,27,28]$, which has shown to be effective in damage identification [29], damage evolution tracking $[27,28,30,31]$ and failure prediction [32] in a nonlinear dynamic system, is investigated. PSW constructs a tracking metric by evaluating the phase space trajectory warping of vibration data measured by sensors, and builds a linear relationship between the tracking metric on the fast time-scale and the actual physical damage on the slow time-scale [32]. As a result, the tracking metric obtained by the PSW can not only monitor the damage evolution in real time, but also serve as input to physics-based models, such as the Paris crack growth model, for bearing failure tracking and RUL prediction. Furthermore, the performance of PSW is enhanced by a multidimensional auto-regression model to improve its damage tracking accuracy, and the Paris crack growth model is modified by a time-piecewise algorithm to meet the real-time computational requirement for RUL prediction.

The rest of the paper is organized as follows. Section II provides the theoretical background and improvement made to enhance the PSW technique. In Section III, a platform for bearing RUL prediction based on a modified Paris crack growth model is introduced. Experimental results, together with related discussions, are presented in Section IV. Finally, conclusions are drawn in Section V.

\section{Enhanced Phase Space Warping}

\section{A. Damage Tracking Based on Multi-Time Scale Modeling}


From the perspective of systems theory, a machine or machine component with an embedded structural defect that evolves over time is viewed as a hierarchical system consisting of a "fast time" subsystem coupled with a "slow time" subsystem, defined as [24-26]:

$$
\begin{aligned}
& \text { Fast time: } \dot{\boldsymbol{x}}=f(\boldsymbol{x}, \mu(\boldsymbol{\phi}), t) \\
& \text { Slow time: } \dot{\boldsymbol{\phi}}=\varepsilon g(\boldsymbol{\phi}, \boldsymbol{x}, t)
\end{aligned}
$$

where $\boldsymbol{x} \in R^{n}$ is a fast-time variable which is observed directly and $\phi \in R^{m}$ is a slow-time variable representing the hidden damage state which is not accessible directly; $f(\bullet)$ and $g(\bullet)$ are the fast-time system function and the slow-time system function, respectively; $t$ is the time and $\mu(\bullet)$ is the function of $\phi ; \varepsilon(0<\varepsilon \square 1)$ is constant, which defines the drift rate of the hidden damage state.

Considering a dynamical system as described by equations (1) and (2), the fast-time state with initial damage state $\phi_{0}$ at the initial time $t_{0}$ is purely a function of $\mu\left(\phi_{0}\right)$ and is represented as

$$
\boldsymbol{x}_{0}=F\left(x_{0}, \mu\left(\phi_{0}\right), t_{0}\right)=F\left(\mu\left(\phi_{0}\right)\right)
$$

where $F(\bullet)$ is a function relating $\boldsymbol{x}$ to $\mu(\phi)$. Then, at time $t\left(t>t_{0}\right)$ the state of the fast subsystem is represented as:

$$
\boldsymbol{x}_{t}=F\left(\mu\left(\phi_{t}\right)\right)
$$

At $\phi_{t}=\phi_{0}$, Taylor expansion of equation (4) is expressed as:

$$
\begin{aligned}
\boldsymbol{x}_{t} & =F\left(\mu\left(\boldsymbol{\phi}_{t}\right)\right) \\
& =F\left(\mu\left(\boldsymbol{\phi}_{0}\right)\right)+\frac{\partial F}{\partial \mu} \frac{\partial \mu}{\partial \boldsymbol{\phi}_{t}}\left(\boldsymbol{\phi}_{t}-\boldsymbol{\phi}_{0}\right)+\mathrm{O}\left(\left\|\boldsymbol{\phi}_{t}-\boldsymbol{\phi}_{0}\right\|^{2}\right)
\end{aligned}
$$

where $\mathrm{O}(\bullet)$ represents the high-order minimum function. The initial damage state $\phi_{0}$ is treated 
as a reference state $\phi_{R}$. Let $t_{0}=t_{R}$, then the damage tracking function between time $t_{R}$ and time $t$ is defined as:

$$
\begin{aligned}
\boldsymbol{e} & \equiv \boldsymbol{x}_{t}-\boldsymbol{x}_{R} \\
& =F\left(\mu\left(\boldsymbol{\phi}_{t}\right)\right)-F\left(\mu\left(\boldsymbol{\phi}_{R}\right)\right) \\
& =\frac{\partial F}{\partial \mu} \frac{\partial \mu}{\partial \boldsymbol{\phi}_{t}}\left(\boldsymbol{\phi}_{t}-\boldsymbol{\phi}_{R}\right)+\mathrm{O}\left(\left\|\boldsymbol{\phi}_{t}-\boldsymbol{\phi}_{R}\right\|^{2}\right)
\end{aligned}
$$

Based on the discussion in references [24] and [27], for a certain system, $\frac{\partial F}{\partial \mu} \frac{\partial \mu}{\partial \phi_{t}}$ and $\mathrm{O}\left(\left\|\phi_{t}-\phi_{R}\right\|^{2}\right)$ is treated as constant values and then the tracking function is simplified as:

$$
\boldsymbol{e}=\boldsymbol{k}\left(\phi_{t}-\phi_{R}\right)+\boldsymbol{c}=\boldsymbol{k} \Delta \boldsymbol{\phi}_{t}+\boldsymbol{c}
$$

where $\boldsymbol{k}=\frac{\partial F}{\partial \mu} \frac{\partial \mu}{\partial \boldsymbol{\phi}_{t}}$ and $\boldsymbol{c}=\mathrm{O}\left(\left\|\boldsymbol{\phi}_{t}-\boldsymbol{\phi}_{R}\right\|^{2}\right) ; \Delta \boldsymbol{\phi}_{t}$ represents the damage state changing from $t_{R}$ to $t$. More detailed derivation procedures is seen in reference [27]. From equation (7), it is seen that there exists a linear relationship between the tracking function $\boldsymbol{e}$ and the hidden damage evolution $\Delta \phi_{t}$. Therefore, a suitable tracking function from the fast-time observed data should be obtained in order to track the hidden damage state evolution in the slow-time scale. In this study, a multi-time scale modeling technique called phase space warping (PSW) is investigated to solve this problem, where the phase space of the fast-time observed data is reconstructed and the trajectory warping of the phase space is evaluated to find a damage tracking metric, which is described in detail below.

\section{Fig. 1. Schematic illustration of multi-time scale modeling}

The basic idea of multi-time scale modeling which combines the fast-time scale and the slow-time scale through an intermediate time scale is shown in Fig. 1. It is assumed that there exists an intermediate time scale which is long compared to the fast dynamics but much short compared to slow dynamics. It should be noted that the drift rate $\varepsilon$ in equation (2) is very 
small so that the change of the slow variable in the intermediate time scale is negligible and the fast subsystem in this time scale is considered approximately stationary. Over the intermediate time scale, the phase space of the initial observed data $\left\{x_{R}(t)\right\}_{t=1}^{N}$ is reconstructed through a time-delay approach based on the Takens embedding theorem [33] as:

$$
\boldsymbol{y}_{R}(n)=\left\{x_{R}(n), x_{R}(n+\tau), \ldots, x_{R}(n+(d-1) \tau)\right\}^{T} \quad n=1, \ldots, N-(d-1) \tau
$$

where $n$ is the vector number in the reconstruction space; $N$ is the total number of the observed data points; $\tau$ is the time delay and $d$ is the embedding dimension in the phase space. These parameters are determined using mutual information [34] and false nearest neighbors [35] techniques, respectively. This phase space $\left\{\boldsymbol{y}_{R}(n)\right\}$ is treated as a reference phase space and vectors in the reference phase space are used to determine a reference model $P^{\tau}$ between $\boldsymbol{y}_{R}(n)$ and $\boldsymbol{y}_{R}(n+\tau)$ :

$$
\boldsymbol{y}_{R}(n+\tau)=P^{\tau}\left(\boldsymbol{y}_{R}(n), \phi_{R}\right)
$$

Using the same reconstruction method, the phase space of other observed data at intermediate time scale $t$ can also be reconstructed as:

$$
\boldsymbol{y}_{t}(n)=\left\{x_{t}(n), x_{t}(n+\tau), \ldots, x_{t}(n+(d-1) \tau)\right\}^{T} \quad n=1, \ldots, N-(d-1) \tau
$$

For each vector $\boldsymbol{y}_{t}(n)$, using the reference model $P^{\tau}$, the predictive vector $\hat{\boldsymbol{y}}_{t}(n+\tau)$ is obtained as:

$$
\hat{\boldsymbol{y}}_{t}(n+\tau)=P^{\tau}\left(\boldsymbol{y}_{t}(n), \phi_{R}\right)
$$

Based on the tracking function definition in equation (6), the damage tracking function for PSW is defined as:

$$
\boldsymbol{e}_{t}(n)=P^{\tau}\left(y_{t}(n), \phi\right)-P^{\tau}\left(y_{t}(n), \phi_{R}\right)
$$


Since $P^{\tau}\left(y_{t}(n), \boldsymbol{\phi}\right)$ is directly available in the phase space, equation (12) is rewritten as:

$$
\begin{aligned}
\boldsymbol{e}_{t}(n) & =\boldsymbol{y}_{t}(n+\tau)-P^{\tau}\left(\boldsymbol{y}_{t}(n), \boldsymbol{\phi}_{R}\right) \\
& =\boldsymbol{y}_{t}(n+\tau)-\hat{\boldsymbol{y}}_{t}(n+\tau)
\end{aligned}
$$

The basic idea of tracking function estimation method is described as Fig. 2. For a vector $\boldsymbol{y}_{t}(n)$ in the phase space, if the damage variable doesn't change, trajectories of $\boldsymbol{y}_{t}(n)$ to $\hat{\boldsymbol{y}}_{t}(n+\tau)$ obtained using the reference model in phase space are shown in dash black line. However, considering the real damage evolution from $\phi_{R}$ to $\phi$, the real trajectories from $\boldsymbol{y}_{t}(n)$ to $\boldsymbol{y}_{t}(n+\tau)$ are warping as shown in dash red line. Consequently, the warping degree of trajectories in the phase space is evaluated by the deviation between $\boldsymbol{y}_{t}(n+\tau)$ and $\hat{\boldsymbol{y}}_{t}(n+\tau)$, and this deviation is used to form the tracking function as equation (13).

\section{Fig. 2. Schematic illustration of tracking function estimation}

\section{B. Enhanced Reference Model based on Multidimensional Autoregression Model}

In traditional PSW [24], the reference model $P^{\tau}$ is constructed using a simple local linear model:

$$
\boldsymbol{y}_{R}(n+\tau)=P^{\tau}\left(y_{R}(n), \phi_{R}\right)=A y_{R}(n)+B
$$

which is an one-order linear model and simply uses one vector to estimate another vector $\tau$ times later. However, in a continuous phase space, one vector should be correlated with several neighbor vectors in the last times. Therefore, the relation between $y_{R}(n+\tau)$ and $\left\{\boldsymbol{y}_{R}(n+\tau-1), \ldots, \boldsymbol{y}_{R}(n)\right\}$ is built and vectors $\left\{\boldsymbol{y}_{R}(n+\tau-1), \ldots, \boldsymbol{y}_{R}(n)\right\}$ are used to estimate vector $\boldsymbol{y}_{R}(n+\tau)$. This multi-order modeling method is more accurate than the simple one-order modeling method. In this study, the multi-order reference model is constructed by the autoregression (AR) model as: 


$$
\begin{aligned}
\boldsymbol{y}_{R}(n+\tau) & =P^{\tau}\left(\boldsymbol{y}_{R}(n+\tau-1), \ldots, \boldsymbol{y}_{R}(n), \boldsymbol{\phi}_{R}\right) \\
& =\boldsymbol{A}_{1} \boldsymbol{y}_{R}(n+\tau-1)+\boldsymbol{A}_{2} \boldsymbol{y}_{R}(n+\tau-2)+\ldots+\boldsymbol{A}_{\tau} \boldsymbol{y}_{R}(n)+\boldsymbol{\varepsilon}_{n} \\
& =\sum_{j=1}^{\tau} \boldsymbol{A}_{j} \boldsymbol{y}_{R}(n+\tau-j)+\boldsymbol{\varepsilon}_{n}
\end{aligned}
$$

where $\boldsymbol{A}_{j} \in R^{d^{*} d}$ is the model parameter and $\varepsilon_{n}$ is the white noise. It is seen that the AR-based reference model can fully take the relationship among several neighbor vectors into consideration so that it will be more beneficial than the simple model shown in equation (14) for describing the properties of the phase space. However, since all variables $\boldsymbol{y}_{R}(n+\tau-j)$ in equation (15) are vectors, which represent a multidimensional AR model and the model parameter estimation method of the traditional one-dimensional time series AR model can not be directly used here. Based on the mathematical inference in references [36] and [37], the least square parameter estimation method for multidimensional AR model is introduced as follows.

Equation (15) is rewritten as:

$$
\left(\begin{array}{c}
\boldsymbol{y}_{R(n+\tau)}(1) \\
\vdots \\
\boldsymbol{y}_{R(n+\tau)}(d)
\end{array}\right)=\left(\begin{array}{c}
\boldsymbol{A}_{1}(1) \\
\vdots \\
\boldsymbol{A}_{1}(d)
\end{array}\right) \boldsymbol{y}_{R}(n+\tau-1)+\left(\begin{array}{c}
\boldsymbol{A}_{2}(1) \\
\vdots \\
\boldsymbol{A}_{2}(d)
\end{array}\right) \boldsymbol{y}_{R}(n+\tau-2)+\ldots+\left(\begin{array}{c}
\boldsymbol{A}_{\tau}(1) \\
\vdots \\
\boldsymbol{A}_{\tau}(d)
\end{array}\right) \boldsymbol{y}_{R}(n)+\left(\begin{array}{c}
\boldsymbol{\varepsilon}_{n}(1) \\
\vdots \\
\boldsymbol{\varepsilon}_{n}(d)
\end{array}\right)
$$

where $\boldsymbol{A}_{j}(i) \in R^{*^{*} d}$. For $i=1,2, \ldots, d$,

$$
\boldsymbol{y}_{R(n+\tau)}(i)=\boldsymbol{A}_{1}(i) \boldsymbol{y}_{R}(n+\tau-1)+\boldsymbol{A}_{2}(i) \boldsymbol{y}_{R}(n+\tau-2)+\ldots+\boldsymbol{A}_{\tau}(i) \boldsymbol{y}_{R}(n)+\boldsymbol{\varepsilon}_{n}(i)
$$

After vector transposition, equation (17) is transformed to

$$
\boldsymbol{y}_{R(n+\tau)}(i)=\left(\boldsymbol{y}_{R}^{T}(n+\tau-1), \ldots, \boldsymbol{y}_{R}^{T}(n)\right)\left(\begin{array}{c}
\boldsymbol{A}_{1}^{T}(i) \\
\vdots \\
\boldsymbol{A}_{\tau}^{T}(i)
\end{array}\right)+\boldsymbol{\varepsilon}_{n}(i)
$$

By choosing 


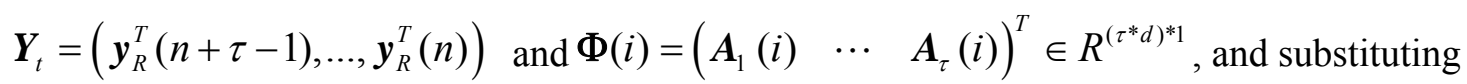
$p$ groups of observed vectors $\left\{\boldsymbol{y}_{R(t+1)}(i) \ldots \boldsymbol{y}_{R(t+p)}(i)\right\}$ into equation (18), it is extended as:

$$
\begin{aligned}
\boldsymbol{y}_{R(t+1)}(i) & =\boldsymbol{Y}_{t} \Phi(i)+\varepsilon_{t}(i) \\
\boldsymbol{y}_{R(t+2)}(i) & =\boldsymbol{Y}_{t+1} \Phi(i)+\varepsilon_{t+1}(i) \\
\vdots & \\
\boldsymbol{y}_{R(t+p)}(i) & =\boldsymbol{Y}_{t+p-1} \Phi(i)+\boldsymbol{\varepsilon}_{t+p-1}(i)
\end{aligned}
$$

Then $\boldsymbol{L}(i), \boldsymbol{B}$ and $\boldsymbol{V}(i)$ are further defined as:

$$
L(i)=\left(\begin{array}{c}
\boldsymbol{y}_{R(t+1)}(i) \\
\vdots \\
\boldsymbol{y}_{R(t+p)}(i)
\end{array}\right), \boldsymbol{B}=\left(\begin{array}{c}
\boldsymbol{Y}_{t} \\
\vdots \\
\boldsymbol{Y}_{t+p-1}
\end{array}\right), \boldsymbol{V}(i)=\left(\begin{array}{c}
\varepsilon_{t}(i) \\
\vdots \\
\boldsymbol{\varepsilon}_{t+p-1}(i)
\end{array}\right)
$$

where $V(i)$ is the residual and $\boldsymbol{V}(i)=\mathbf{L}(i)-\boldsymbol{B} \Phi(i)$. To estimate the optimal model parameter, the residual error should be as small as possible. This is achieved by minimizing the following objective function:

$$
\boldsymbol{J}(i)=\boldsymbol{V}^{T}(i) \boldsymbol{V}(i)
$$

To minimize the objective function, the least square estimation of model parameters is calculated as:

$$
\hat{\boldsymbol{\Phi}}(i)=\left(\boldsymbol{B}^{T} \boldsymbol{B}\right)^{-1} \boldsymbol{B}^{T} \boldsymbol{L}(i), \quad i=1,2 \ldots, d
$$

and the least square estimation of white noise deviation is obtained as:

$$
\hat{\varepsilon}_{n}(i)=\frac{1}{p}(\boldsymbol{L}(i)-\boldsymbol{B} \hat{\Phi}(i))^{T}(\boldsymbol{L}(i)-\boldsymbol{B} \hat{\Phi}(i)), \quad i=1,2 \ldots, d
$$

It should be note that $\boldsymbol{B} \in R^{p \times(\tau \times d)}$ and $\boldsymbol{B}^{T} \boldsymbol{B} \in R^{(\tau \times d) \times(\tau \times d)}$. According to the matrix theory:

$$
\mathrm{r}\left(\boldsymbol{B}^{T} \boldsymbol{B}\right) \leq \mathrm{r}(\boldsymbol{B}) \leq \min (p, \tau \times d)
$$

where $\mathrm{r}(\bullet)$ is the rank of the objective matrix. To ensure the full rank and invertibility of $\boldsymbol{B}^{T} \boldsymbol{B}, p$ should be larger than $(\tau \times d)$. In this study, $p$ is chosen as $p>2(\tau \times d)$ to confirm 
the effectiveness of equation (22).

\section{Tracking Metric Estimation and Enhanced PSW Algorithm}

With the enhanced reference model, we can further compute the tracking function shown in equation (13). For a vector $\boldsymbol{y}_{t}(n)$ in the phase space, we will find $p$ nearest neighbor vectors, which are evaluated by the distance from $\boldsymbol{y}_{t}(n)$ to vectors in the reference phase space, to construct the AR-based reference model and then compute the tracking function for this vector as:

$$
\begin{aligned}
\boldsymbol{e}_{t}(n) & =\boldsymbol{y}_{t}(n+\tau)-\hat{\boldsymbol{y}}_{t}(n+\tau) \\
& =\boldsymbol{y}_{t}(n+\tau)-\left(\left(\boldsymbol{y}_{t}^{T}(n+\tau-1), \quad \cdots \quad, \boldsymbol{y}_{t}^{T}(n)\right)(\hat{\boldsymbol{\Phi}}(1), \quad \cdots \quad, \hat{\boldsymbol{\Phi}}(d))\right)^{T}-\hat{\boldsymbol{\varepsilon}}_{n}
\end{aligned}
$$

For $M=N-(d-1) \tau$ vectors in the phase space, we obtain $M$ tracking functions in total and they are combined together using the root mean square method $[24,27]$ to get a tracking metric for the phase space at time $t$. The tracking metric is defined as:

$$
\boldsymbol{e}_{t}=\sqrt{\frac{\sum_{n=1}^{M} q(n)\left\|\boldsymbol{e}_{t}(n)\right\|^{2}}{\sum_{n=1}^{M} q(n)}}
$$

where $q(n)$ is the weight function which is used to adjust the contribution degree of each $\boldsymbol{e}_{t}(n)$ to the final tracking metric. It should be noted that the $p$ nearest neighbor vectors which are found to construct the enhanced reference model have a severe impact on $\boldsymbol{e}_{t}(n)$ calculation. The smaller the region composed by the $p$ nearest neighboring points is, the more accurate both the reference model and the tracking function are expected to be. Therefore, the tracking function obtained using small neighbor region should own high contribution degree to the final tracking metric and the weight function $q(n)$, which can quantitatively describe the contribution degree, is defined as [27]: 


$$
q(n)=\frac{1}{r_{n}^{m}}
$$

where $r_{n}$ is the distance from the vector $\boldsymbol{y}_{t}(n)$ to the farthest of all $p$ nearest neighbors and $m$ is the correlation dimension of this phase space. From this equation, it is seen that the vector with small neighbor region will have large weight value and on the contrary, the vector with large neighbor region will have small weight value.

By combining the traditional PSW with the enhanced AR-based reference model, the procedures of enhanced PSW algorithm as shown in Fig. 3 is summarized as follows:

(1) First, the sample rate is treated as the fast-time scale and the sample time of a data set is treated as the intermediate-time scale. The sample interval between two data sets is considered as the slow-time scale and the whole bearing lifetime is considered as the composition of various slow-time scales.

(2) At the initial time $t=0$, the phase space of data set 0 is reconstructed and used as the reference phase space.

(3) At time 1, phase space 1 of data set 1 is reconstructed using the same time delay and dimension as the reference phase space. For each vector in phase space $1, p$ nearest neighbor vectors in the reference phase space are searched and the AR-based reference model is constructed to obtain the tracking function for each vector, respectively. Then the tracking metric $\boldsymbol{e}_{1}$ at time 1 is computed using equation (26).

(4) Following the same procedure as in (3), the tracking metric for each intermediate-time scale is computed to track the hidden damage of a bearing.

Fig. 3. Flowchart of enhanced PSW for damage tracking 


\section{RUL Prediction Based on Modified Paris Crack Growth Model}

\section{A. Modified Paris Crack Growth Model}

As the PSW is able to build a linear relationship between the tracking metric extracted from the raw sensor signal and the hidden physical damage variable, the metric is utilized as an input to a physics-based model for bearing RUL prediction. Paris law is a well-known principle that has been widely used to guide crack growth detection and prediction in mechanical materials [38-40]. In 1963, Paris [41] proposed an empirical crack growth model based on the stress intensity factor (SIF) as:

$$
\frac{d l}{d N}=C_{0}(\Delta K)^{n}
$$

where $l$ is the crack length, $N$ is the fatigue life, $\Delta K$ represents the amplitude of the SIF, $C_{0}$ and $n$ are material constants. Based on the crack growth equation, the RUL is obtained as:

$$
N_{R U L}=N_{t h}-N_{i}=\int_{l_{i}}^{l_{t h}} \frac{d l}{C_{0}(\Delta K)^{n}}
$$

where $N_{t h}$ is whole lifetime and $N_{i}$ is the current life. In addition, $l_{t h}$ and $l_{i}$ are the crack failure threshold and the current crack length, respectively. For ball bearings, the SIF is estimated as [38]:

$$
\Delta K=\tau_{\max } \sqrt{\pi l} Y
$$

where $\tau_{\max }$ is the maximum shear stress and $Y$ is the geometric correction factor which is related to the crack shape. Furthermore, based on the PSW theory introduced in section II, there exists a linear relationship between the tracking metric $\boldsymbol{e}_{t}$ obtained by the enhanced PSW and the real damage variable (i.e. the crack length in this study). Therefore, it is assumed that: 


$$
e_{t}=k l
$$

where $k$ is a constant coefficient. By substituting equation (30) and (31) into equation (29), we can yield:

$$
\begin{aligned}
N_{R U L} & =\int_{e_{i}}^{e_{t h}} \frac{k^{2} d e_{t}}{C_{0} \tau_{\max }^{n} Y^{n} \pi^{n / 2} k^{n / 2}\left(e_{t}\right)^{n / 2}} \\
& =\int_{e_{i}}^{e_{t h}} \frac{k^{2}}{C_{0} \tau_{\max }^{n} Y^{n} \pi^{n / 2} k^{n / 2}} \frac{d e_{t}}{\left(e_{t}\right)^{n / 2}}
\end{aligned}
$$

where $e_{i}$ is the current tracking metric and $e_{t h}$ is a predefined failure threshold. For commonly-used bearing materials, $n$ is chosen as 2 and $C_{0}, Y, k$ and $\tau_{\max }$ are all constants.

Taking these conditions into consideration, equation (32) is rewritten as:

$$
N_{R U L}=\int_{e_{i}}^{e_{t h}} \frac{k^{2}}{C_{0} \tau_{\max }^{2} Y^{2} \pi k} \frac{d e_{t}}{e_{t}}=D \int_{e_{i}}^{e_{t h}} \frac{d e_{t}}{e_{t}}=D \ln \frac{e_{t h}}{e_{i}}+D_{0}
$$

where $D=\frac{k^{2}}{C_{0} \tau_{\max }^{2} Y^{2} \pi k}$ and $D_{0}$ is the integral constant. Equation (33) represents the predicted RUL based on traditional Paris crack growth model. The RUL is computed only using the tracking metric at current time and the threshold metric. However, in the initial crack growth stage, the crack growth rate is so slow that the variation of the tracking metric is not very significant. In addition, since the tracking metric is calculated from sensor signals, it is vulnerable to noise, such that the tracking metric can not keep increasing in the initial crack growth stage. If the RUL is solely determined by $e_{i}$, there will be large prediction errors generated for $N_{R U L}$. Therefore, for $D$ and $D_{0}$ in equation (33), a piecewise time-modified strategy is introduced in this study. Specifically, it defines $t_{\text {initial }}$ as the starting time of the initial crack growth stage, and $e_{\text {initial }}$ is the corresponding tracking metric at the time $t_{\text {initial. }}$. For any damage tracking metric $e_{i}$ in the initial crack growth stage, the RUL is determined as: 


$$
N_{R U L}=D_{1} \ln \frac{e_{t h}}{e_{i}}-\left(t_{i}-t_{\text {initial }}\right)\left(1-\frac{e_{i}-e_{\text {initial }}}{e_{\text {initial }}}\right)
$$

where $D_{1}$ is the constant which is determined by the historical data collected in the initial crack growth stage. It is seen from equation (34) that a $e_{i}$ is approaching $e_{\text {initial }}$, the temporal correlation $\left(t_{i}-t_{\text {initial }}\right)\left(1-\frac{e_{i}-e_{\text {initial }}}{e_{\text {initial }}}\right)$ is approximately equal to $t_{i}-t_{\text {initial }}$ so that error of life prediction will be removed from $D_{1} \ln \frac{e_{t h}}{e_{i}}$. Furthermore, it is noted that, with an increased $e_{i}$, the term $\left(t_{i}-t_{\text {initial }}\right)\left(1-\frac{e_{i}-e_{\text {initial }}}{e_{\text {initial }}}\right)$ will become smaller than $t_{i}-t_{\text {initial }}$, and the effect of the time correlation will gradually decrease. This means that after the crack initiation stage, crack propagation will enter a stage where its growth will accelerate due to material fatigue caused by repetitive loading as bearing operates. This will be reflected in a significant increase of $e_{i}$, which means that the effect of temporal correlation is no longer significant, and the RUL at this stage is estimated as:

$$
N_{R U L}=D_{2} \ln \frac{e_{t h}}{e_{i}}
$$

where $D_{2}$ is determined by the data collected during the fast crack growth stage. Combining equation (34) with (35), the modified Paris crack growth model is summarized as follows:

$$
N_{R U L}= \begin{cases}D_{1} \ln \frac{e_{t h}}{e_{i}}-\left(t_{i}-t_{\text {initial }}\right)\left(1-\frac{e_{i}-e_{\text {initial }}}{e_{\text {initial }}}\right), & e_{i} \leq \alpha e_{\text {initial }} \\ D_{2} \ln \frac{e_{t h}}{e_{i}}, & e_{i}>\alpha e_{\text {initial }}\end{cases}
$$

where $\alpha e_{\text {initial }}$ is used as the threshold to distinguish the fast crack growth stage from the initial crack growth stage. 


\section{B. Simulation Test}

To numerically evaluate the effectiveness of the modified Paris crack growth model proposed above, a simulation test is conducted first. A piecewise function is constructed as equation (37) to simulate the tracking metric obtained by the PSW for tracking the bearing crack growth process [42]:

$$
e= \begin{cases}\frac{1}{100}(0.002 t+1)+0.001 * \text { randn, } & t \leq 200 \\ \frac{1}{100 b}\left(1.04{ }^{t}\right)+0.001 * \text { randn }, & t>200\end{cases}
$$

For the numerical study, the term $b$ is set as 1821.96 to ensure the continuity of the signal and the symbol randn represents the white noise. Equation (37a) is a linear function for simulating the initial crack growth stage and equation (37b) is an exponential function to simulate the fast crack growth stage. It should be noted that, since the tracking metric calculated from sensor signals is not always keeping increasing, white noise is added into the simulation signal. The simulated bearing degradation is shown in Fig. 4. There are 200 points for the initial crack growth stage and 50 points for the fast crack growth stage. The first point is treated as $e_{\text {initial }}=0.0127$ and the last point is treated as $e_{t h}=0.998$. First, traditional Paris crack growth model is used to predict the RUL for these 250 points and the results are shown in Fig. 5. It is seen that the predicted RUL curve only follows the changing trend of the degradation curve, but cannot well track the changing trend of the real RUL curve. Then, a threshold-adjustment Paris crack growth model designed as equation (38) is used to predict the RUL [38]:

$$
N_{R U L}=\int_{e_{i}}^{\lambda e_{t h}} \frac{k^{2}}{C_{0} \tau_{\max }^{2} Y^{2} \pi k} \frac{d e_{t}}{e_{t}}=D \ln \frac{\lambda e_{t h}}{e_{i}}
$$


where the threshold coefficient $\lambda$ is brought in to adjust the failure threshold in traditional Paris model. In this study, this method sets $0.8 e_{t h}($ i.e. $\lambda=0.8)$ as the failure threshold for the initial crack growth stage and sets $e_{t h}($ i.e. $\lambda=1)$ as the failure threshold for the fast crack growth stage. Then equation (38) is used for RUL prediction and the results are shown in Fig. 6. Although the predicted RUL curve can basically track the changing trend of the real RUL curve, there exists large fluctuation in the curve, resulting in large prediction error. At last, the modified Paris crack growth model proposed in this paper is utilized for RUL prediction and the results are shown in Fig. 7. Particularly, $D_{1}$ and $D_{2}$ are set as 120.8 and 25.3, respectively. It is seen from Fig. 7 that the RUL prediction curve obtained by the modified Paris crack growth model can not only track the changing trend of the real RUL curve well, but also has small fluctuation and prediction error. The results of the simulation study demonstrate that the modified Paris crack growth model is able to estimate RUL precisely based on the simulated bearing degradation curve. Thus it is used for bearing RUL prediction.

Fig. 4. Simulated bearing degradation process

Fig. 5. RUL prediction using traditional Paris crack growth model Fig. 6. RUL prediction using threshold-adjustment Paris crack growth model Fig. 7. RUL prediction using modified Paris crack growth model

\section{Multi-Time Modeling Framework for RUL Prediction}

Integrating the modified Paris crack growth model with the tracking metric obtained by the PSW, a multi-time scale modeling approach for bearing failure tracking and RUL prediction is designed as shown in Fig. 8. The enhanced PSW method is firstly used to compute tracking metric $e_{t}$ using sensor data at current time. Once $e_{t}>e_{\text {initial, }}$, the bearing is suffered from the 
initial defect and the tracking metric is used as the input to the modified Paris crack growth model for RUL prediction. By comparing $e_{t}$ and $\alpha e_{\text {initial }}$, the current bearing crack growth stage is determined and the initial crack growth prediction equation (36-1) or the fast crack growth prediction (36-2) will be chosen to predict the bearing RUL at this time. Then, as time increases, the RUL prediction will be conducted continuously until the tracking metric reaches the predefined failure threshold.

\section{Fig. 8. Flowchart of multi-time scale modeling algorithm}

\section{Experimental Study}

To verify the effectiveness of the proposed bearing RUL prediction approach based on the enhanced PSW and modified Paris crack growth model, vibration signals from two run-to-failure experiments contributed by NSF I/UCR Center on Intelligent Maintenance Systems [43] are analyzed. The test system in the experiments is shown in Fig. 9(a) and the structure of the system is shown in Fig. 9(b). The test system was run constantly at $2000 \mathrm{rpm}$ and $6000 \mathrm{lb}$ radial load was placed onto the shaft and bearing through a spring mechanism. Four double row bearings (Bearing 1-4, model: ZA-2115) were installed on the shaft and all the bearings were lubricated with an oil circulation system that regulated the flow and the temperature of the lubricant. A magnetic plug installed in the oil feedback pipe was used to collect debris from the oil as evidence of bearing degradation. When the accumulated debris adhered to the magnetic plug exceeded a certain level, an electrical switch turned off and the test stopped. The vibration signals are collected by accelerometers (Model: PCB 353B33 High Sensitivity Quartz ICP) installed on each bearing. Vibration signals of bearings were collected every 10 minutes. The sampling rate is $20 \mathrm{kHz}$ and each data file includes 20480 
points.

The first run-to-failure test is conducted from 10:32, February 12, 2004 to 6:22, February 19, 2004. At the end of the test, an outer race defect occurs in bearing 1 as shown in Figure 10(a). There are one-channel signals, 984 data files, corresponding to bearing 1 in this test. The second run-to-failure test is conducted from 14:39, October 29, 2003 to 23:39, November 25, 2003. At the end of the test, an inner race defect occurred in bearing 3 as shown in Fig. 10(b). There are two-channel signals (X direction and Y direction), 2000 data files, corresponding to bearing 3 in this test. Due to the same bearing model and experiment conditions, $\mathrm{X}$-direction signal of bearing 3 in test 2 is treated as the historical data for these two tests.

Fig. 9. Experimental system for bearing run-to-failure test [44]

Fig. 10. Defective bearing used for run-to-failure experiment [43]

\section{A. Case Study I}

Vibration signals from bearing 1 in test 1 are analyzed in this case. Each data file is set as observed data in the intermediate-time scale. The first data file is used to construct the reference phase space with time delay $\tau=3$ and dimension $d=5$. As it is discussed in section II (B), $p$ should be larger than $2\left(\tau^{*} d\right) .32$ nearest neighbor vectors from the reference phase space are chosen to form the AR-based reference model. Using the enhanced PSW algorithm, 983 tracking metrics are calculated and bearing degradation curve are shown in Fig. 11. Using the Chebyshev inequality proposed in our previous work [10], the initial failure threshold $e_{\text {initial }}$ is set as 0.0811 and the initial failure time starts at the $586^{\text {th }}$ point. After the initial failure point, the degradation curve increases slowly at first and has a significant increase trend at the end of the test, which is consistent with the process of bearing failure evolution. To make a comparison, we use the traditional PSW and root mean square (RMS) to 
compute the tracking metrics and the degradation curves are shown in Fig. 12 and Fig. 13, respectively. In Fig. 12, there exists large fluctuation in the bearing degradation area so that failure evolution trend cannot be seen clearly in the degradation curve. In Fig. 13, although RMS-based degradation curve has a similar increasing trend with the curve formed by the enhanced PSW, the fluctuation in the bearing degradation area is also very large, which may severely affect the accuracy of the RUL prediction. The comparison results indicate that the AR-based reference model construction method can significantly improve the effectiveness and accuracy for bearing damage tracking. Furthermore, the tracking metrics obtained by the enhanced PSW have less fluctuation and can display a clearer trend in bearing degradation progress than that obtained by traditional PSW and RMS.

Fig. 11. Degradation curve using enhanced PSW: bearing 1

\section{Fig. 12. Degradation curve using traditional PSW: bearing 1}

Fig. 13. Degradation curve using RMS: bearing 1

For the bearing with small size such as ZA-2115, when the crack size evolves more than 3 times the initial crack size, catastrophic accidents is considered to be eminent, and this crack size is chosen as the failure threshold. It should be noted that linear relationship exists between the real physical damage variables (i.e. crack size in this case) and the tracking metric obtained by the PSW. Accordingly in this study, the failure threshold for the tracking metric was chosen as $e_{\mathrm{th}}=3 e_{\text {initial }}=0.245$, as shown in Fig. 11. Based on the modified Paris crack growth model introduced in section III, RUL prediction starts at the initial failure point. Given that $e_{\mathrm{th}}=3 e_{\text {initial}}, \alpha e_{\text {initial }}$ in equation (35) was chosen as $\alpha e_{\text {initial }}=0.5 e_{\mathrm{th}}=1.5 e_{\text {initial }}$ in this study to distinguish the initial crack growth stage with the fast crack growth stage. Furthermore, $D_{1}$ and $D_{2}$ in equation (35) are set as 273 and 42 using the historical data. They are determined as follows: $\quad$ Using the proposed enhanced PSW method, the tracking metric of historical data in the whole bearing life time was obtained. Then the $e_{\text {initial }}$ and 
$e_{\mathrm{th}}=3 e_{\text {initial }}$ was computed, and their corresponding RUL values of $e_{\text {initial }}, 1.5 e_{\text {initial }}$ and

$e_{\mathrm{th}}=3 e_{\text {initial }}$ were determined from the real experiment results. At last, the value of $e_{\text {initial }}$, $1.5 e_{\mathrm{initial}}$ and $e_{\mathrm{th}}$ and their RUL values were substituted into equation (36-1) and (36-2) to compute the values of $D_{1}$ and $D_{2}$. The prediction results are shown in Fig. 14, where the horizontal ordinate represents current prediction time and the vertical ordinate represents corresponding predicted RUL. It is seen that the predicted RUL values can well track the changing trend of the real bearing RUL values. The predicted RUL value matches the real RUL value as the prediction gets closer to the failure point. The observed fluctuation near the $940^{\text {th }}$ point is attributed to the switching of the tracking metrics to the fast crack-growth stage. For purpose of comparison, RUL predictions using the traditional Paris crack growth model and threshold-adjusted Paris crack growth model were performed, and the results are shown in Figs. 15 and 16, respectively. It is seen from Fig. 15 that at the initial crack growth stage, the predicted RUL values cannot reliably track the trend of the real RUL using the traditional Paris crack growth model. While the threshold-adjusted method has shown to improve the prediction accuracy at the initial crack growth stage, significant fluctuation in the predicted RUL curve is illustrated in Fig. 16. To further evaluate the performance of the developed method with other integrated data-driven and physics-based techniques, particle filter integrated with the Paris model is comparatively studied for bearing RUL prediction. In this method, the RMS values shown in Fig. 13 are used as the tracking metric and the Paris model is treated as the state model, which is defined as:

$$
\begin{aligned}
& N_{R U L}(k)=\frac{\ln \left(R M S_{t h} / R M S_{k}\right)}{\ln \left(R M S_{t h} / R M S_{k-1}\right)} N_{R U L}(k-1)+\omega_{k-1} \\
& N_{R U L, z}(k)=N_{R U L}(k)+v_{k}
\end{aligned}
$$

In Eq. (39), $N_{R U L}(k)$ is the RUL at time $k, N_{R U L, z}(k)$ represents the observed value of $N_{R U L}(k)$, $\omega_{k-1}$ is the process noise at time $k-1$, and $v_{k}$ is the measurement noise at time $k$. The result of RUL prediction by particle filter are shown in Fig. 17. While this method has shown to track 
the general trend of the realistic RUL values, the predicted RUL in Fig. 17 continues to show significant fluctuation as compared to the result in Fig. 14. The prediction error is quantitatively evaluated by the average error and the root mean square error using the following equations:

$$
\begin{aligned}
& e_{1}=\frac{1}{M} \sum_{i=1}^{M}\left|R U L_{p}(i)-R U L_{r}(i)\right| \\
& e_{2}=\sqrt{\frac{1}{M} \sum_{i=1}^{M}\left(R U L_{p}(i)-R U L_{r}(i)\right)^{2}}
\end{aligned}
$$

where $R U L_{p}$ is the predicted RUL value, $R U L_{r}$ is the real RUL value, and $M=382$ is the number of predicted RUL values. The prediction error for each method is shown in Table 1:

Table 1. Comparison of prediction error by different methods: bearing 1

It can also be seen from Table 1 that the prediction error obtained by the modified Paris crack growth model is much smaller than those obtained by the traditional model, the threshold-adjusted model, and the traditional model integrated with particle filter. The results indicate that the time-piecewise modified strategy is more effective than the rest of the methods in increasing the accuracy and reliability of bearing RUL prediction.

Fig. 14. RUL prediction using modified Paris crack growth model: bearing 1

Fig. 15. RUL prediction using traditional Paris crack growth model: bearing 1

Fig. 16. RUL prediction using threshold-adjusted Paris crack growth model: bearing 1 Fig. 17. RUL prediction using Paris crack growth model integrated with particle filter:

\section{bearing 1}

\section{B. Case Study II}

Vibration signals ( $\mathrm{Y}$ direction) from bearing 3 in test 2 are analyzed in this case to confirm the above analysis results. The first data file is used to construct the reference phase space with time delay $\tau=3$ and dimension $d=5$, and 32 nearest neighbor vectors from the reference 
phase space are chosen to form the AR-based reference model. Using the enhanced PSW algorithm, 2000 tracking metrics are calculated and bearing degradation curve is shown in Fig. 18. The initial failure threshold $e_{\text {initial }}$ is set as 0.1544 and the initial failure time starts at the $1808^{\text {th }}$ point. After the initial failure point, the degradation curve also increases slowly at first and then has a significant increase trend at the end of the test, which is consistent with the process of bearing failure evolution. To make a comparison, we also use the traditional PSW and root mean square (RMS) to compute the tracking metrics and draw degradation curves in Figs. 19 and 20, respectively. Although degradation curves obtained by these two methods can display the similar increasing trend with the curve formed by the enhanced PSW, the fluctuation in the bearing degradation area in Figs. 19 and 20 is larger than that in Fig. 18 when they are observed under the same coordinate range from 0.1 to 0.6 . The comparison results confirm the effectiveness of the AR-based reference model construction method for improving tracking accuracy and further indicate that the enhanced PSW are more beneficial than traditional PSW and RMS for bearing damage tracking.

Fig. 18. Degradation curve using enhanced PSW: bearing 3

Fig. 19. Degradation curve using traditional PSW: bearing 3

Fig. 20. Degradation curve using RMS: bearing 3

Using the same method as case study I, RUL prediction using the modified Paris crack growth model is conducted for bearing 3 . The failure threshold is determined as $e_{\mathrm{th}}=0.4656$ and $\alpha e_{\text {initial }}$ is also chosen as $\alpha e_{\text {initial }}=0.5 e_{\mathrm{th}}=1.5 e_{\text {initial }}$ to distinguish the initial crack growth stage from the fast crack growth stage. Furthermore, $D_{1}$ and $D_{2}$ in equation (35) are the same as that in case study I. The prediction results are shown in Fig. 21. It is seen that the predicted RUL values can also well fit the changing trend of the real bearing RUL values. Furthermore, RUL prediction using the traditional Paris crack growth model, threshold-adjusted Paris crack growth model, and particle filter integrated with the Paris model are investigated, with the 
corresponding results shown in Figs. 22, 23 and 24, respectively. Using the same equations as those for case study I, the prediction error for each method is computed and listed in Table 2 . Compared with the traditional and threshold-adjusted Paris model, RUL prediction using the modified Paris crack growth model has returned significantly smaller prediction error. In addition, while the prediction error associated with the integrated particle filter and Paris model is smaller than that of both the traditional and threshold-adjusted Paris models, it is larger than that of the proposed PSW and Paris crack growth model. The results further demonstrates the effectiveness of the proposed multi-time scale method for system degradation prediction.

Table 2. Comparison of prediction error by different methods: bearing 3

Fig. 21. RUL prediction using modified Paris crack growth model: bearing 3

Fig. 22. RUL prediction using traditional Paris crack growth model: bearing 3

Fig. 23. RUL prediction using threshold-adjustment Paris crack growth model: bearing 3

Fig. 24. RUL prediction using Paris crack growth model integrated with particle filter: bearing 3

\section{Conclusions}

A multi-time scale modeling approach combining an enhanced PSW with a modified Paris crack growth model to bearing failure tracking and RUL prediction is presented. This method builds a linear relationship between the tracking metric obtained from real-time vibration sensor data and actual physical damage variables of bearings, and make full use of both data-driven method (i.e., enhanced PSW) and physics-based method (i.e., modified Paris crack growth model) for RUL prediction. Experimental study on two run-to-failure bearing experiments indicates that AR-based method for reference model construction can significantly improve the accuracy of PSW in damage tracking. The modified Paris crack growth model has shown to effectively estimate the RUL of rolling bearings with good 
reliability. The proposed technique demonstrates the effectiveness of a new approach to analyzing damage propagation in a dynamical system by constructing two complementary models that address both the fast and slow time-scale aspects of the system behavior, therefore improving the RUL prediction.

\section{Acknowledgement}

This work has been supported in part by the National Natural Science Foundation of China (51175080), Scientific Research Foundation of Graduate School of Southeast University (YBJJ1424), Postgraduate Research \& Innovation Project of Jiangsu Province, and the Fundamental Research Funds for the Central Universities (CXZZ12-0096). The last author acknowledges funding from the National Science Foundation under award CMMI-1300999, which has supported his work on system diagnosis and prognosis.

\section{References}

[1] A. Heng, S. Zhang, A. Tan, J. Mathew, Rotating machinery prognostics: state of the art, challenges and opportunities. Mechanical Systems and Signal Processing 23(3) (2009) $724-739$.

[2] A. Malhi, R. Yan, R. X. Gao, Prognosis of defect propagation based on recurrent neural networks. IEEE Transactions on Instrumentation and Measurement 60(3) (2011) 703-711.

[3] Y. Pan, J. Chen, L. Guo, Robust bearing performance degradation assessment method based on improved wavelet packet- support vector data description. Mechanical Systems and Signal Processing 23(3) (2009) 669-681.

[4] D. He, R. Li, J. Zhu, Plastic bearing fault diagnosis based on a two-step data mining approach. IEEE Transactions on Industrial Electronics 60(8) (2013) 3429-3440.

[5] J. Yu, Local and nonlocal preserving projection for bearing defect classification and performance assessment. IEEE Transactions on Industrial Electronics 59(5) (2012) 2363-2376. 
[6] J. Yu, Bearing performance degradation assessment using locality preserving projections and Gaussian mixture models. Mechanical System and Signal Processing 25(7) (2011) 2573-2588.

[7] J. Yu, Health condition monitoring of machines based on hidden Markov model and contribution analysis. IEEE Transactions on Instrumentation and Measurement 61(8) (2012) 2200-2211.

[8] F. Cong, J. Chen,Y. Pan, Kolmogorov-Smirnov test for rolling bearing performance degradation assessment and prognosis. Journal of vibration and control 17(9) (2010) $1337-1347$.

[9] N. Gebraeel, M. Lawley, R. Liu, V. Parmeshwaran, Residual life predictions from vibration-based degradation signals: a neural network approach. IEEE Transactions on Industrial Electronics 51(3) (2004) 694-700.

[10]Y. Qian, R. Yan and S. Hu, Bearing degradation evaluation using recurrence quantification analysis and Kalman filter, IEEE Transactions on Instrumentation and Measurement 63(11) (2014) 2599-2610.

[11]R. Singleton, E. Strangas, S. Aviyente, Extended Kalman filtering for remaining-useful-life estimation of bearings. IEEE Transactions on Industrial Electronics 62(3) (2015) 1781-1790.

[12]C. Chen, B. Zhang and G. Vachtsevanos, Prediction of machine health condition using neuro-fuzzy and Bayesian algorithm. IEEE Transactions on Instrumentation and Measurement 61(2) (2012) 297-306.

[13]C. Chen, B. Zhang, G. Vachtsevanos, M. Orchard, Machine condition prediction based on adaptive neuro-fuzzy and high-order particle filtering. IEEE Transactions on Industrial Electronics 58(9) (2011) 4353-4364.

[14]M. Yu, D. Wang, M. Luo, An integrated approach to prognosis of hybrid systems with unknown mode changes. IEEE Transactions on Industrial Electronics 62(1) (2015) $503-515$. 
[15]P. Wang and R. Gao, Adaptive resampling-based particle filtering for tool life prediction. Journal of Manufacturing Process 37(2) (2015) 528-534.

[16] J. Wang, P. Wang, and R. Gao, Enhanced particle filter for tool wear prediction. Journal of Manufacturing Systems 36 (2015) 35-45.

[17] M. Yaqub, I. Gondal, J. Kamruzzaman, Multi-step support vector regression and optimally parameterized wavelet packet transform for machine residual life prediction. Journal of Vibration and Control 19(7) (2013) 963-974.

[18] A. Soualhi, H. Razik, G. Clerc, D. Doan, Prognosis of bearing failures using hidden markov models and the adaptive neuro-fuzzy inference system. IEEE Transactions on Industrial Electronics 61(6) (2014) 2864-2874.

[19] Y. Li, S. Billington, C. Zhang, T. Kurfess, S. Danyluk, S. Liang, Adaptive prognostics for rolling element bearing condition. Mechanical Systems and Signal Processing 13(1) (1999) 103-113.

[20] Y. Li, T.R. Kurfess, S.Y. Liang, Stochastic prognostics for rolling element bearings. Mechanical Systems and Signal Processing 14(5) (2000) 747-762.

[21] Y. Shih, J. Chen, Analysis of fatigue crack growth on a cracked shaft. International Journal of Fatigue 19(6) (1997) 477-485.

[22] C.H. Oppenheimer, K.A. Loparo, Physically based diagnosis and prognosis of cracked rotor shafts. Proceedings of SPIE Component and Systems Diagnostics, Prognostics, and Health Management II, Bellingham America, pp. 122-132, 2002.

[23] Y. Choi, C. Liu, Spall progression life model for rolling contact verified by finish hard machined surfaces. Wear 262(1-2) (2007) 24-35.

[24] D. Chelidze, A nonlinear observer for damage evolution tracking. $\mathrm{PhD}$ Thesis, The Pennsylvania State University, 2000.

[25]S. Sheng, Multi-time scale modeling strategy for bearing life prognosis. PhD Thesis, University of Massachusetts, Amherst, 2008.

[26] S. Sheng and R. Gao, Multi-time scale modeling strategy for bearing life prognosis. 
Proceedings of the 2009 ASME Dynamic Systems and Control Conference, pp. 645-652, Hollywood, CA, October 12-14, 2009.

[27] D. Chelidze, J. P. Cusumano, A. Chatterjee, 'Dynamical systems approach to damage evolution tracking, part 1: description and experimental application. Journal of Vibration and Acoustics. 124(2) (2002) 250-257.

[28] J. P. Cusumano, D. Chelidze, A. Chatterjee, Dynamical systems approach to damage evolution tracking, part 2: model-based validation and physical interpretation. Journal of Vibration and Acoustics 124(2) (2002) 258-264.

[29] D. Chelidze, Identifying multidimensional damage in a hierarchical dynamical system. Nonlinear Dynamics 37 (2004) 307-322.

[30] J. P. Cusumano, A. Chatterjee, Steps towards a qualitative dynamics of damage evolution. International Journal of Solids and Structures 37(44) (2000) 6397-6417.

[31] B. Fan, L. Hu, N. Hu, Fault tracking of rotating machinery under variable operation based on phase space warping. Acta Physica Sinica $\quad$ 62(16) (2013) 160503.

[32] D. Chelidze, J. P. Cusumano, A dynamical systems approach to failure prognosis. Journal of Vibration and Acoustics 126(1) (2004) 2-8.

[33] F. Takens, Detecting strange attractors in turbulence. Dynamical Systems and Turbulence, Lecture Notes in Mathematics, Rand, D. A. and Young, L.S (Eds.), Springer-Verlag, Berlin, pp. 366-381, 1981.

[34] A. M. Fraser, H. L. Swinney, Independent coordinates for strange attractors from mutual information. Physical Review A 33 (1986) 1134-1140.

[35] M. B. Kennel, R. Brown, H. D. Abarbanel, Determining embedding dimension for phase-space reconstruction using a geometrical construction. Physical Review A 45(6) (1992) 3403-3411.

[36] G. Pap, M. VanZuijlen, Asymptotic inference for nearly unstable multidimensional AR processes. Theory of Probability and its Applications 41(3) (1997) 578-586.

[37] C. Zhang, Multi-dimensional AR series modeled by least square criterion. Geomatics 
and Information Science of Wuhan University 27(4) (2002) 377-381.

[38] D. Xu, J. Huang, Q. Zhu, Residual fatigue life prediction of ball bearings based on paris law and RMS. Chinese Journal of Mechanical Engineering 25(2) (2012) 320-327.

[39] N. Pugno, M. Ciavarella, P. Cornetti, A generalized Paris' law for fatigue crack growth. Journal of the Mechanics and Physics of Solids 54(7) (2006) 1333-1349.

[40] M. L. Cohen, S. S. Kulkarni, J. D. Achenbach, Probabilistic approach to growth and detection of a truncated distribution of initial crack lengths based on Paris' law. Structural Health Monitoring 1(2) (2012) 225-236.

[41] P. C. Paris, F. Erdogan, A critical analysis of crack propagation laws. Journal of Basic Engineering 85(4) (1963) 528-534.

[42] T. Goshima, S. Ishihar, M. Shimizu, Crack propagation and initiation lives for surface pitting due to rolling/sliding contact. Journal of Thermal Stresses 33(11) (2010) 1087-1106.

[43] H. Qiu, J. Lee, J. Lin, G. Yu, Robust performance degradation assessment methods for enhanced rolling element bearings prognostics. Advanced Engineering Informatics 17(. 3-4) (2003) 127-140.

[44] H. Qiu, J. Lee, J. Lin, Wavelet filter-based weak signature detection method and its application on roller bearing prognostics. Journal of Sound and Vibration 289(4-5) (2006) 1066-1090. 


\section{Figure Lists}

Fig. 1. Schematic illustration of multi-time scale modeling

Fig. 2. Schematic illustration of tracking function estimation

Fig. 3. Flowchart of enhanced PSW for damage tracking

Fig. 4. Simulated bearing degradation process

Fig. 5. RUL prediction using traditional Paris crack growth model

Fig. 6. RUL prediction using threshold-adjustment Paris crack growth model

Fig. 7. RUL prediction using modified Paris crack growth model

Fig. 8. Flowchart of multi-time scale modeling algorithm

Fig. 9. Experimental system for bearing run-to-failure test [44]

Fig. 10. Defective bearing used for run-to-failure experiment [43]

Fig. 11. Degradation curve using enhanced PSW: bearing 1

Fig. 12. Degradation curve using traditional PSW: bearing 1

Fig. 13. Degradation curve using RMS: bearing 1

Fig. 14. RUL prediction using modified Paris crack growth model: bearing 1

Fig. 15. RUL prediction using traditional Paris crack growth model: bearing 1

Fig. 16. RUL prediction using threshold-adjustment Paris crack growth model: bearing 1

Fig. 17. RUL prediction using Paris crack growth model integrated with particle filter: bearing 1

Fig. 18. Degradation curve using enhanced PSW: bearing 3

Fig. 19. Degradation curve using traditional PSW: bearing 3

Fig. 20. Degradation curve using RMS: bearing 3

Fig. 21. RUL prediction using modified Paris crack growth model: bearing 3

Fig. 22. RUL prediction using traditional Paris crack growth model: bearing 3

Fig. 23. RUL prediction using threshold-adjustment Paris crack growth model: bearing 3

Fig. 24. RUL prediction using Paris crack growth model integrated with particle filter: bearing 3 
Table 1. Comparison of prediction error by different methods: bearing 1

\begin{tabular}{ccccc}
\hline & $\begin{array}{c}\text { Modified Paris } \\
\text { crack growth } \\
\text { model }\end{array}$ & $\begin{array}{c}\text { Traditional Paris } \\
\text { crack growth } \\
\text { model }\end{array}$ & $\begin{array}{c}\text { Threshold-adjusted } \\
\text { Paris' crack growth } \\
\text { model }\end{array}$ & $\begin{array}{c}\text { Particle filter and } \\
\text { Paris crack growth } \\
\text { model }\end{array}$ \\
\hline $\begin{array}{c}\text { Prediction error } \\
e_{1} \text { (unit: hour) }\end{array}$ & 4.61 & 18.32 & 7.09 & 7.60 \\
\hline $\begin{array}{c}\text { Prediction error } \\
e_{2} \text { (unit: hour) }\end{array}$ & 5.15 & 22.80 & 9.12 & 9.12 \\
\hline
\end{tabular}


Table 2. Comparison of prediction error by different methods: bearing 3

\begin{tabular}{ccccc}
\hline & $\begin{array}{c}\text { Modified Paris } \\
\text { crack growth } \\
\text { model }\end{array}$ & $\begin{array}{c}\text { Traditional Paris } \\
\text { crack growth } \\
\text { model }\end{array}$ & $\begin{array}{c}\text { Threshold-adjusted } \\
\text { Paris' crack growth } \\
\text { model }\end{array}$ & $\begin{array}{c}\text { Particle filter and } \\
\text { Paris crack growth } \\
\text { model }\end{array}$ \\
\hline $\begin{array}{c}\text { Prediction } \text { error } \\
e_{1} \text { (unit: hour) }\end{array}$ & 0.98 & 19.31 & 11.35 & 5.58 \\
\hline $\begin{array}{c}\text { Prediction } \text { error } \\
e_{2} \text { (unit: hour) }\end{array}$ & 1.15 & 22.65 & 14.29 & 7.9 \\
\hline
\end{tabular}




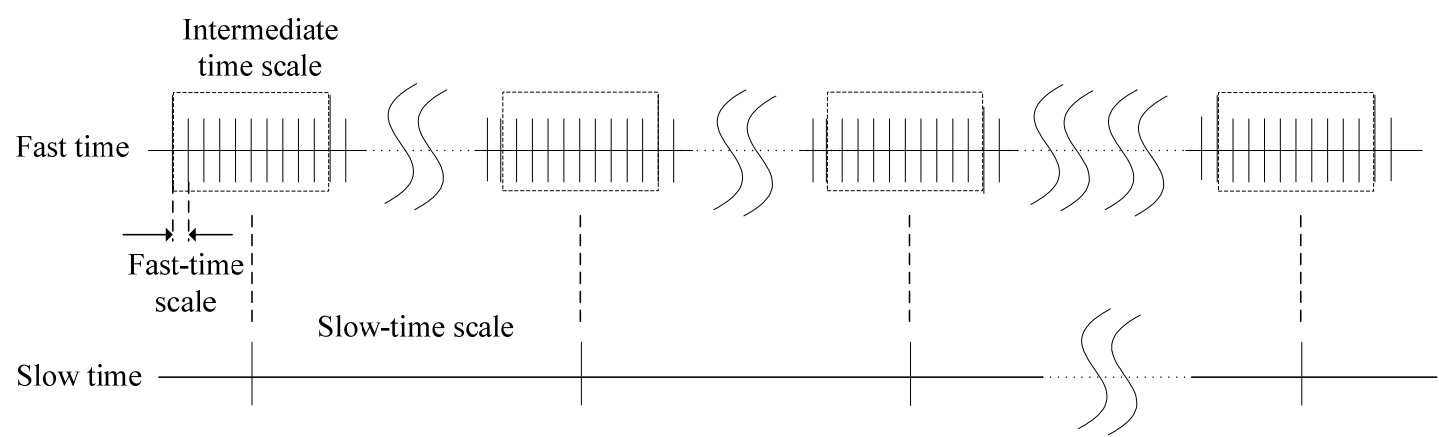

Fig.1. Schematic illustration of multi-time scale modeling 


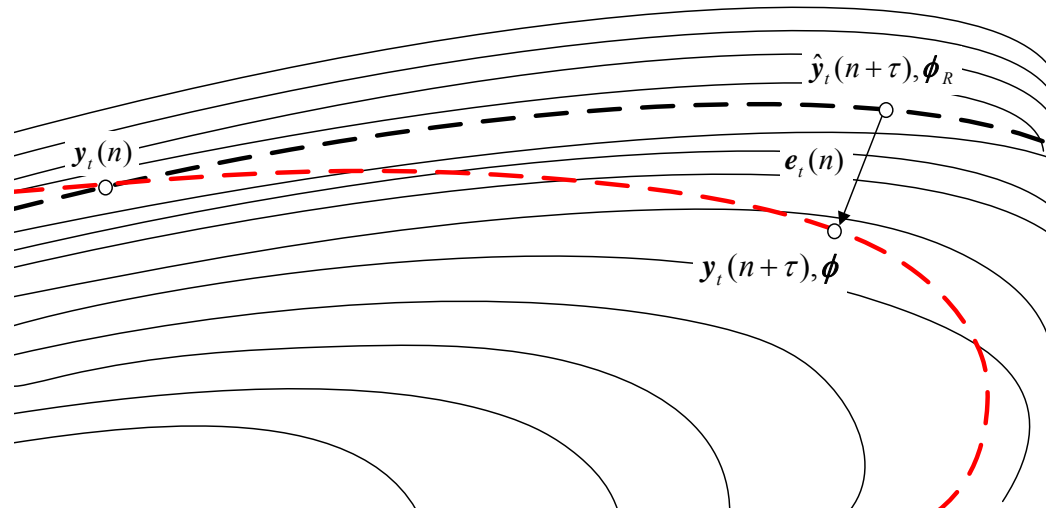

Fig.2. Schematic illustration of tracking function estimation 
Bearing lifetime data

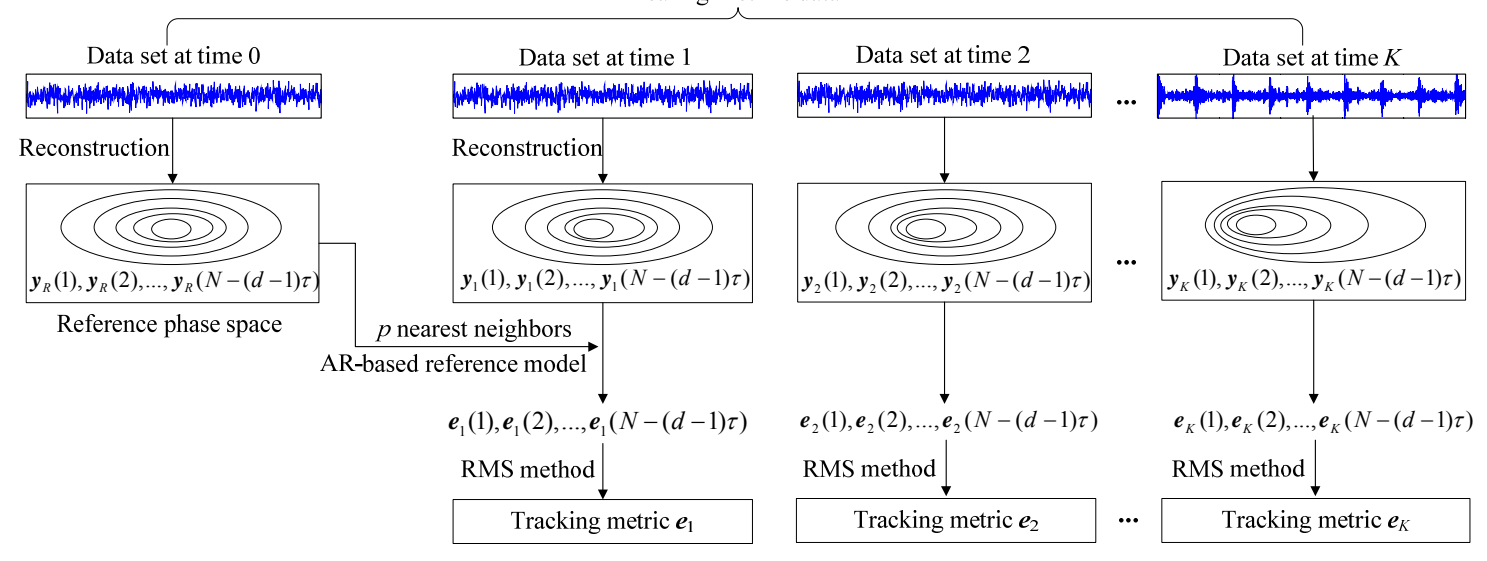

Fig. 3. Flowchart of enhanced PSW for damage tracking 


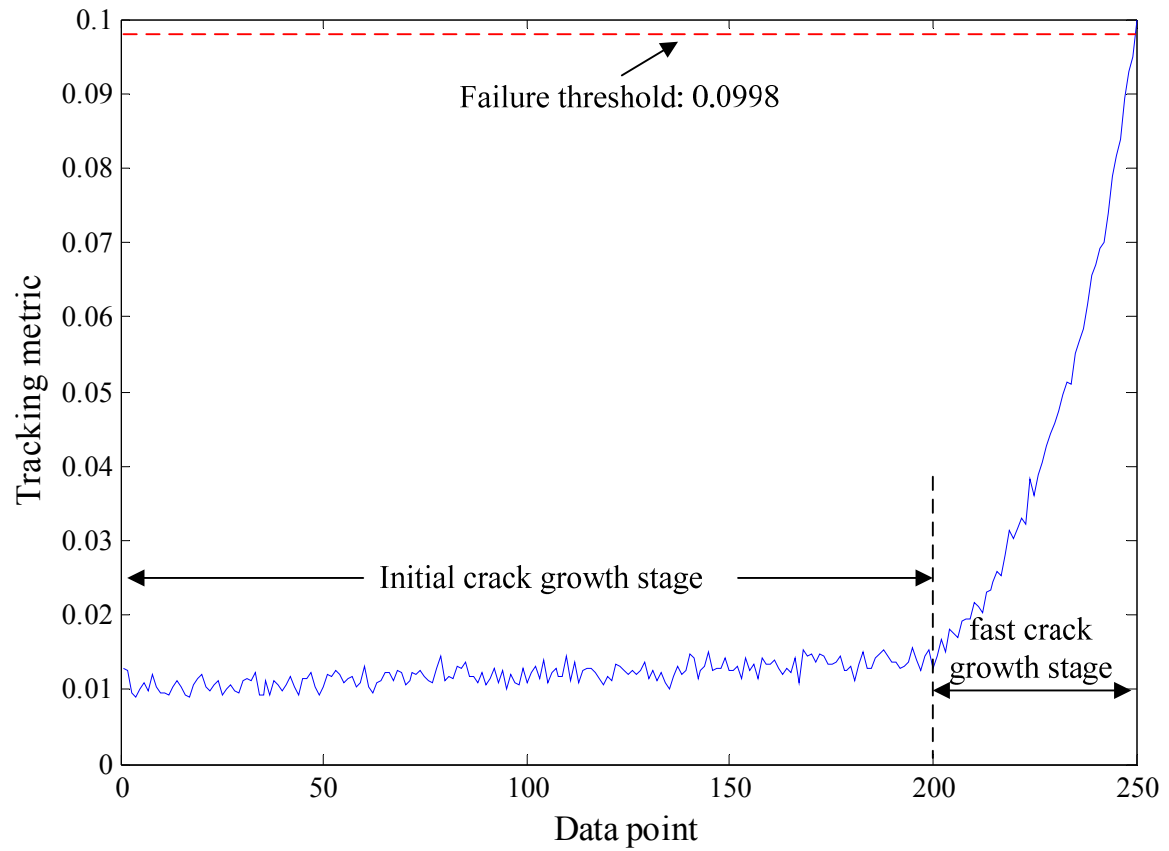

Fig. 4. Simulated bearing degradation process 


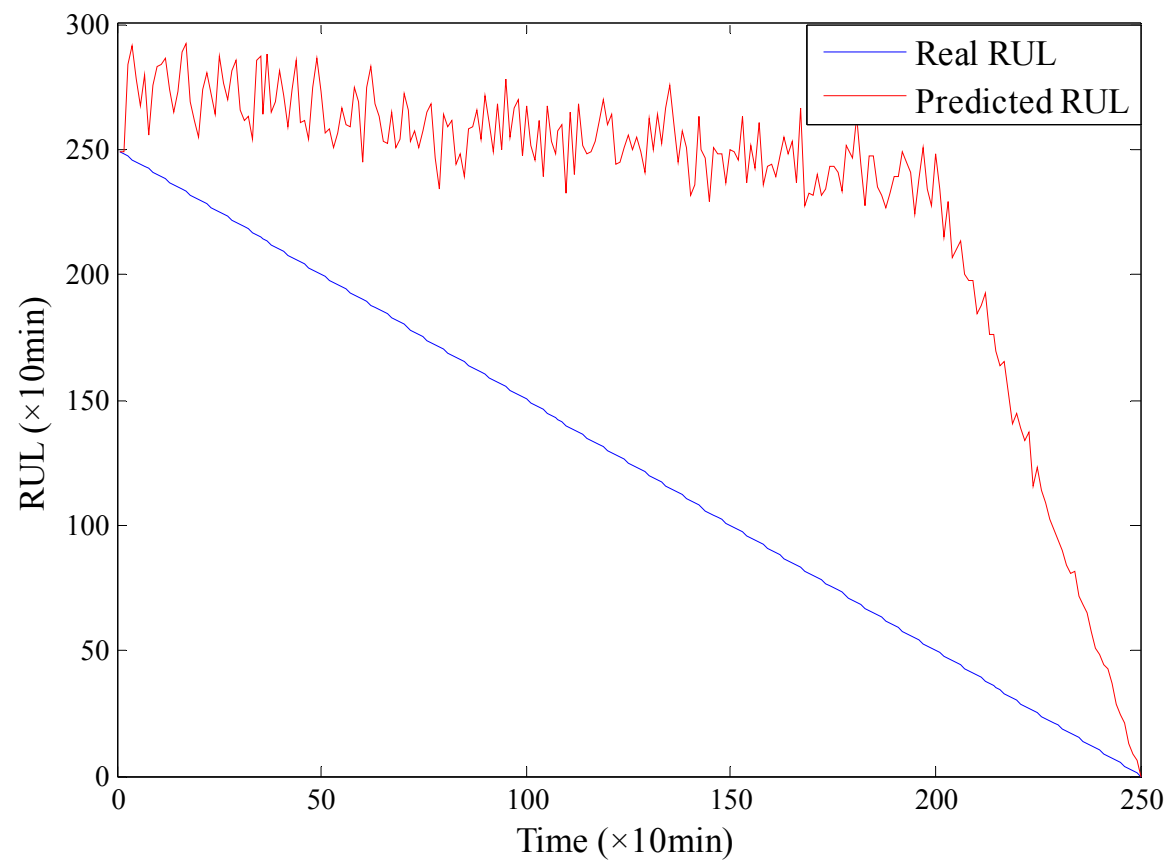

Fig. 5. RUL prediction using traditional Paris crack growth model 


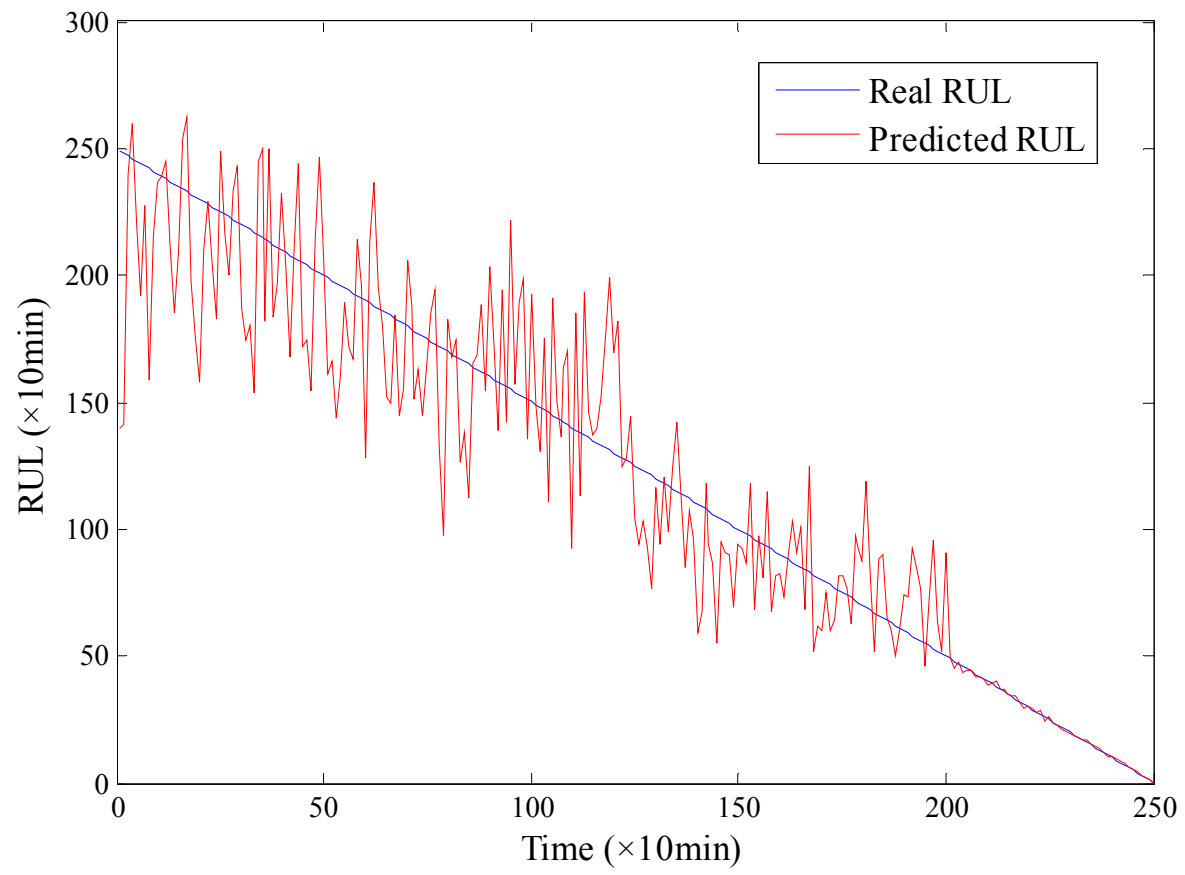

Fig. 6. RUL prediction using threshold-adjusted Paris crack growth model 


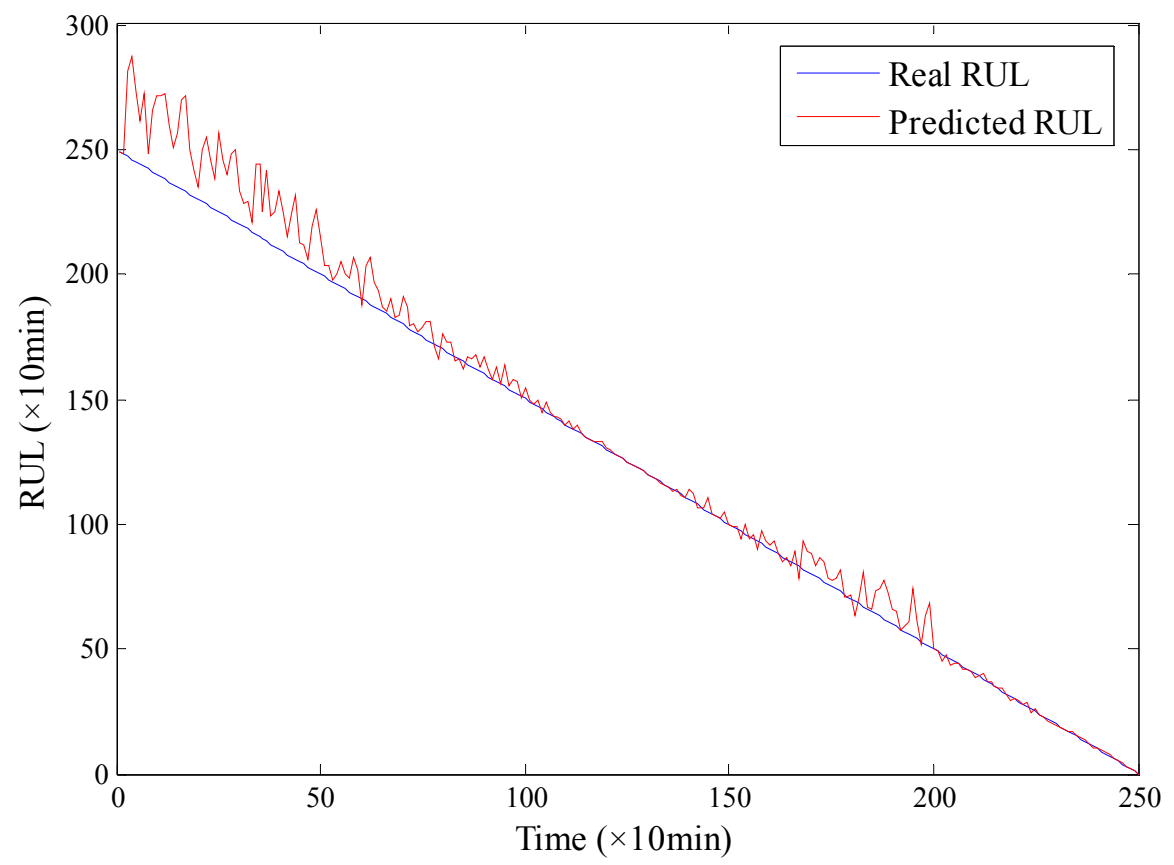

Fig. 7. RUL prediction using modified Paris crack growth model 


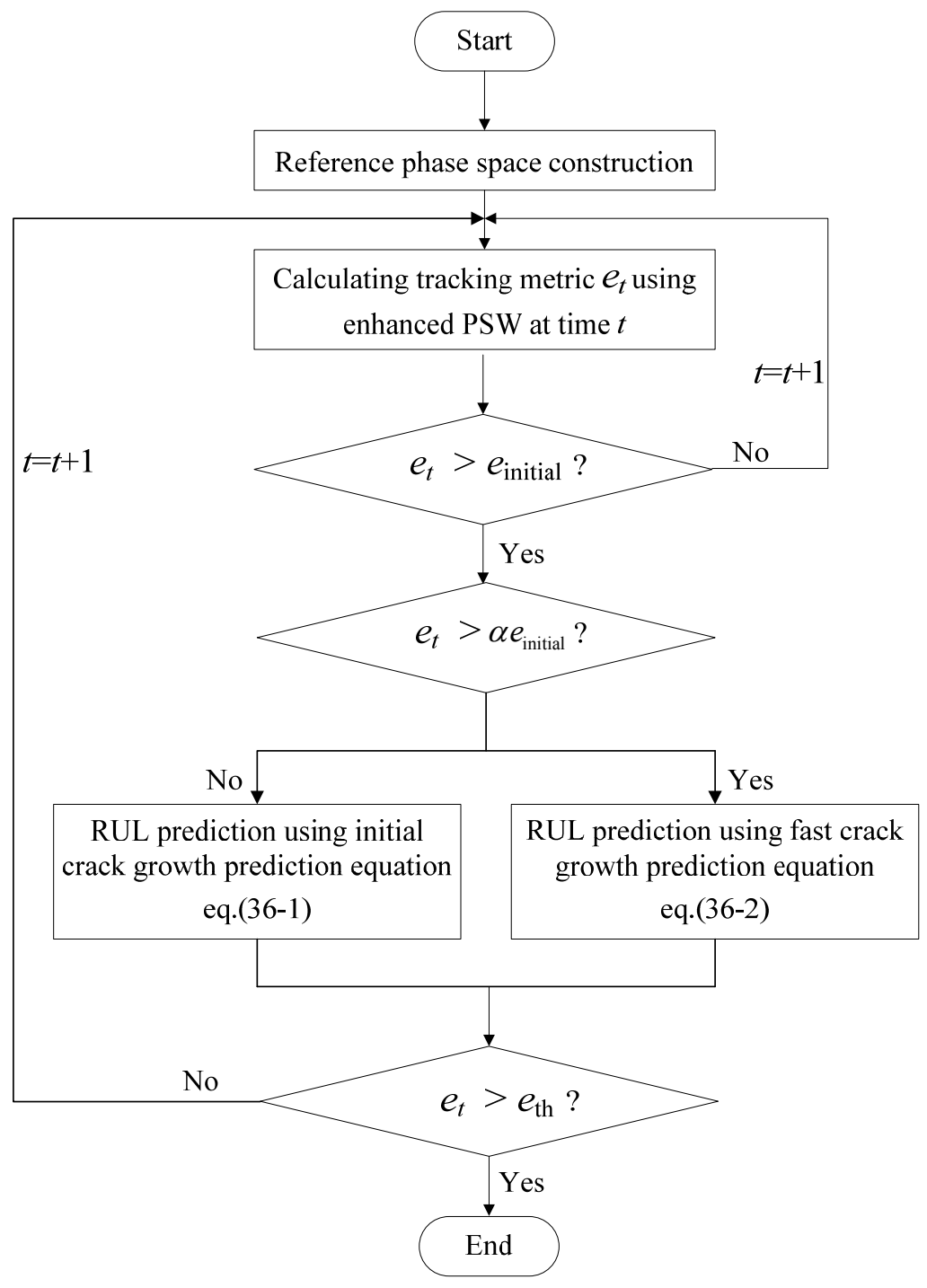

Fig. 8. Flowchart of multi-time scale modeling algorithm 


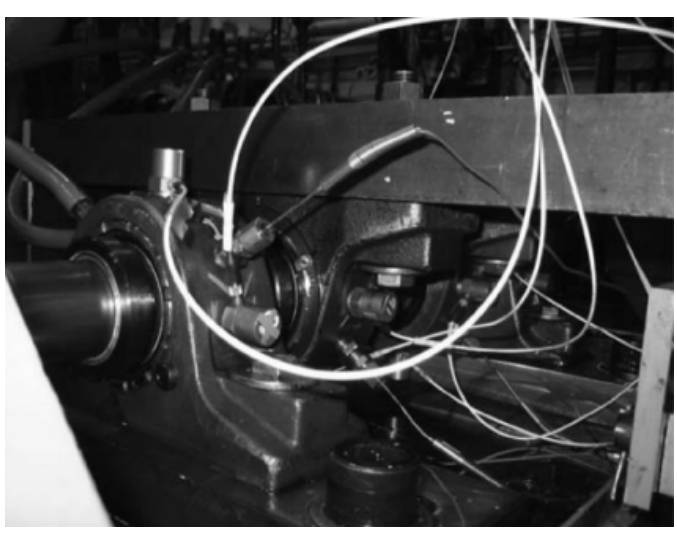

(a) Test system

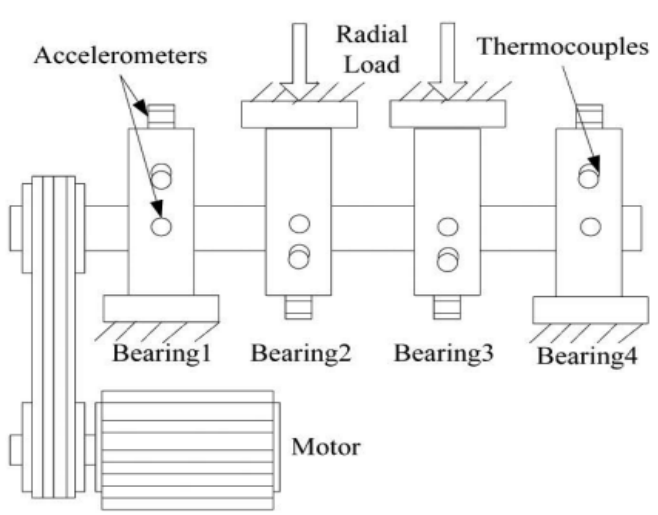

(b) System structure

Fig. 9. Experimental system for bearing run-to-failure test [44] 


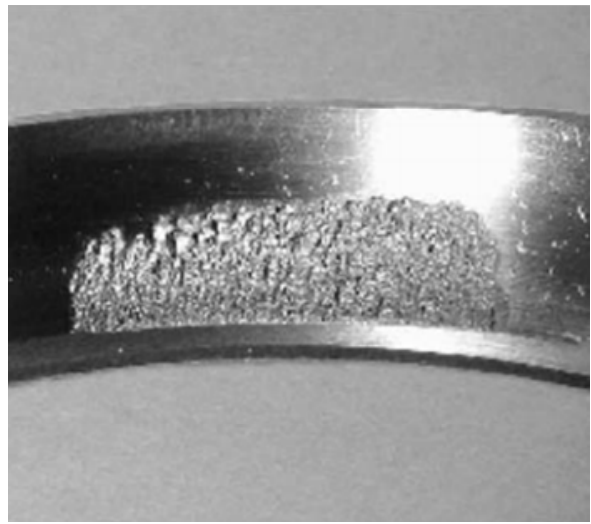

(a) Outer race defect in bearing 1

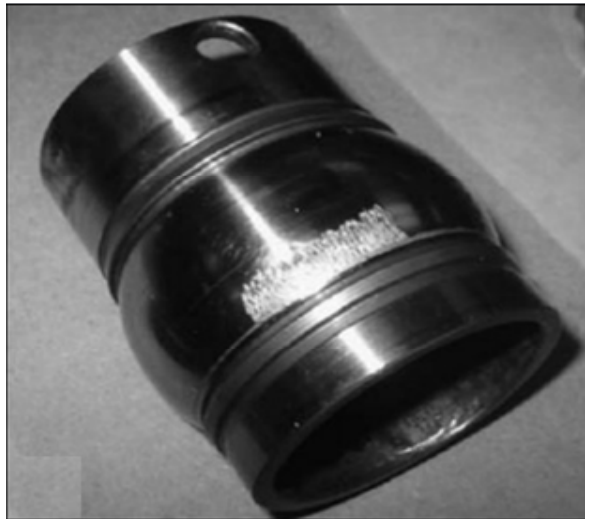

(b) Inner race defect in bearing 3

Fig. 10. Defective bearing used for run-to-failure experiment [43] 


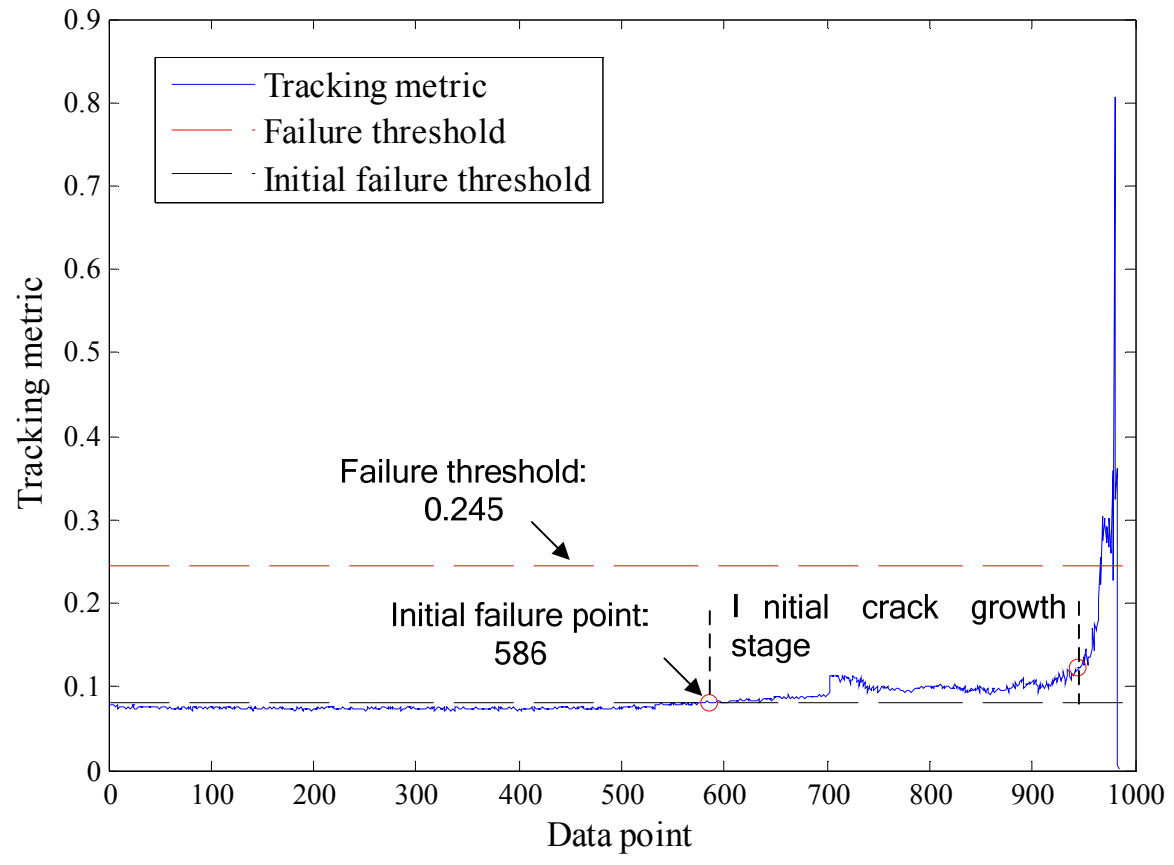

Fig.11. Degradation curve using enhanced PSW: bearing 1 


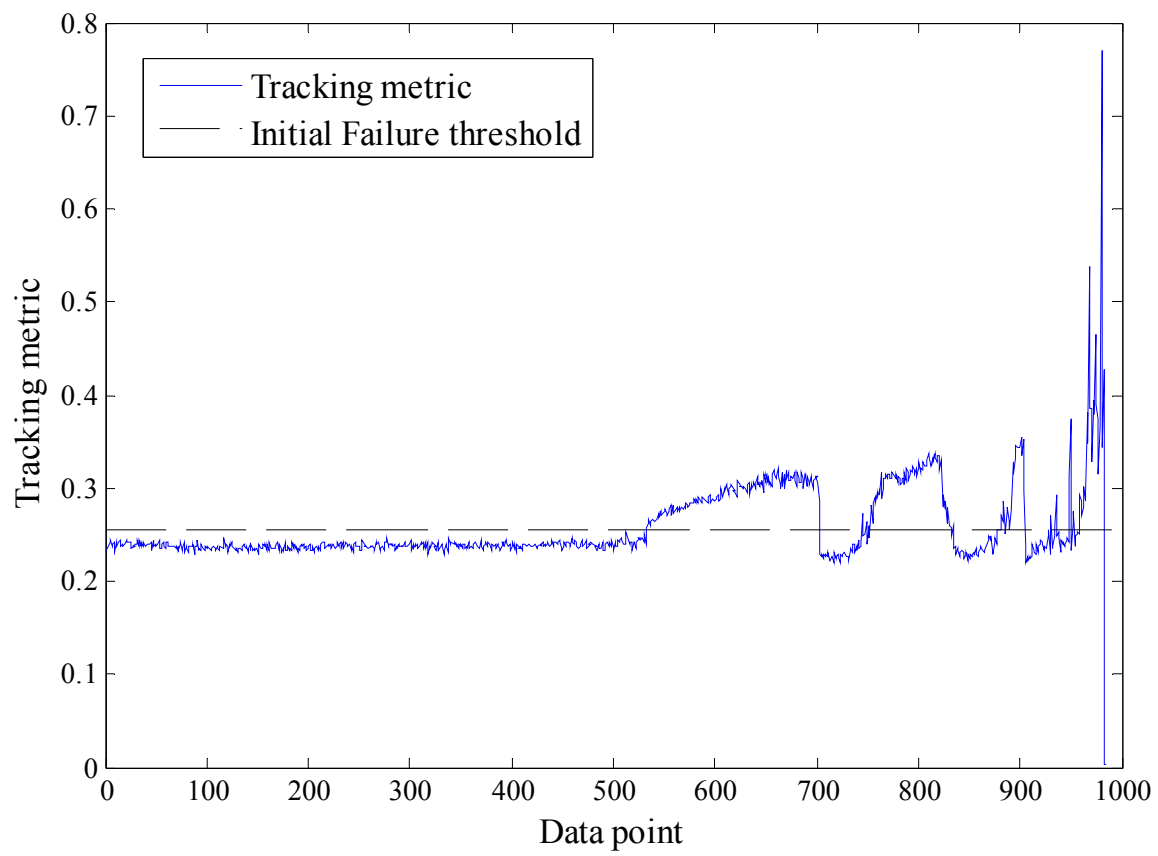

Fig. 12. Degradation curve using traditional PSW: bearing 1 


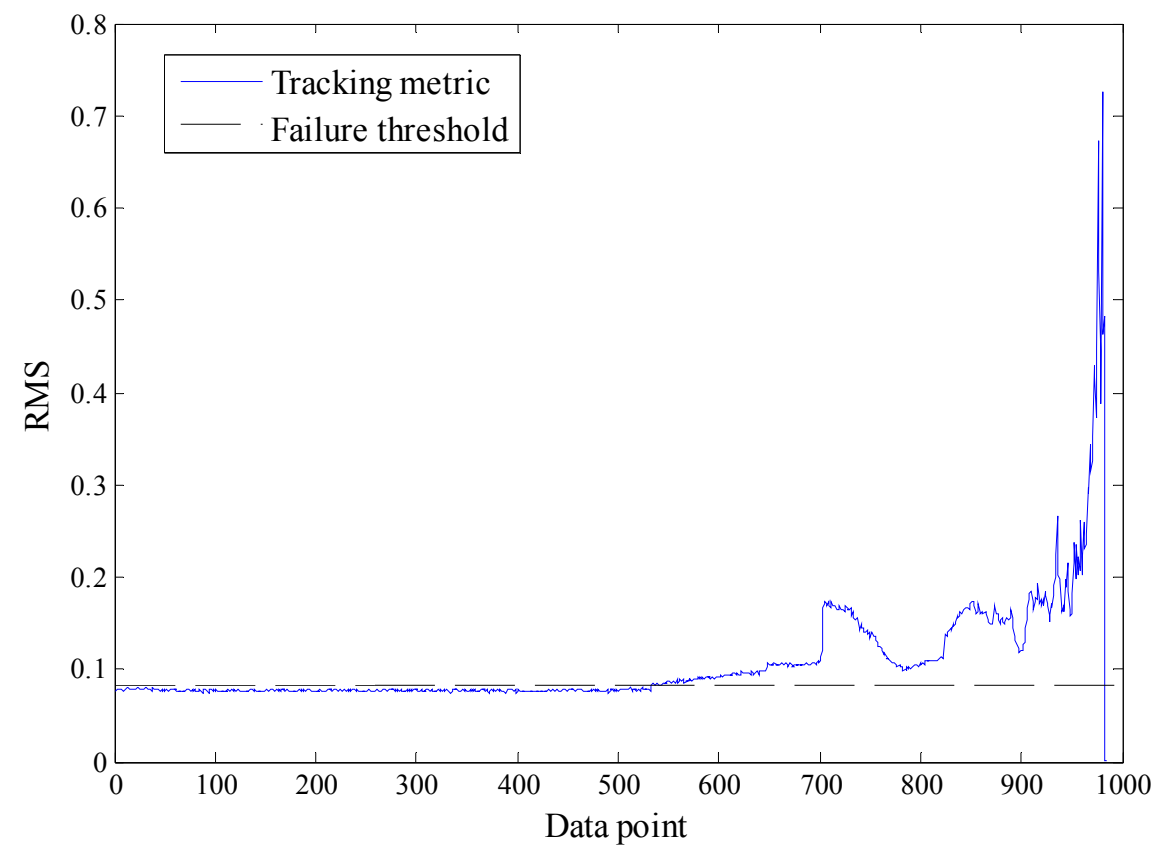

Fig.13. Degradation curve using RMS: bearing 1 


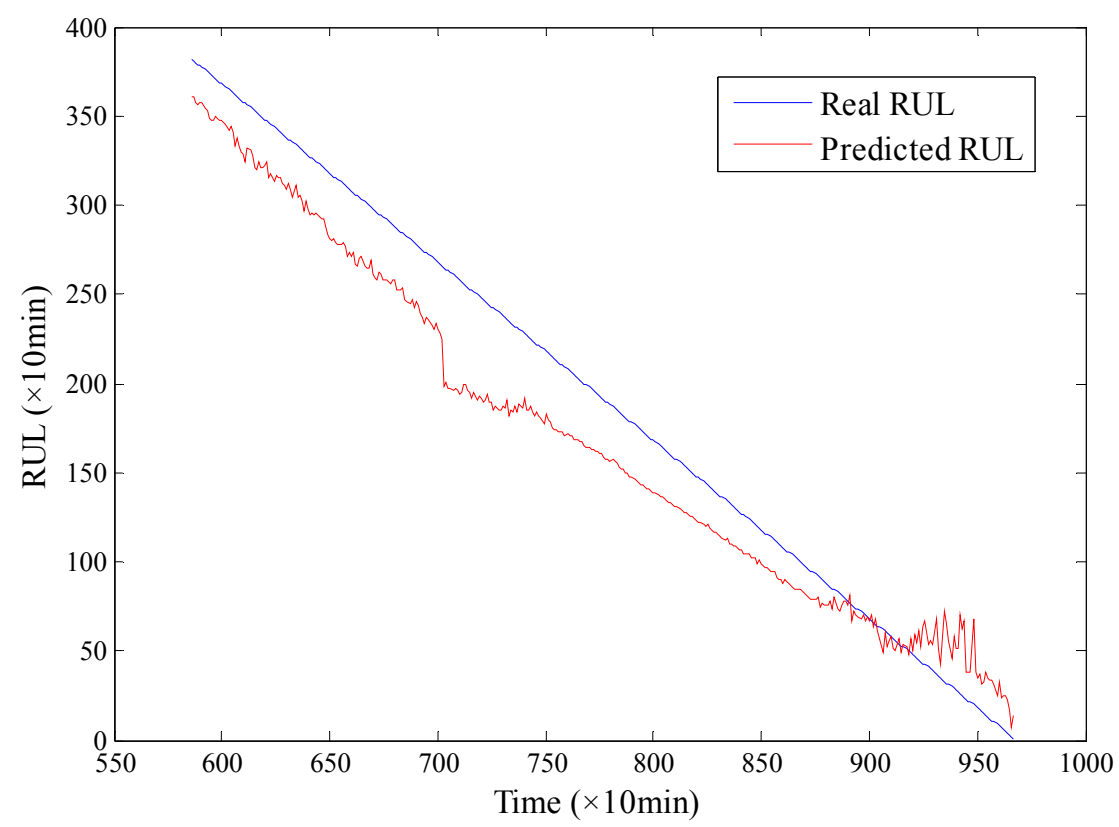

Fig.14. RUL prediction using modified Paris crack growth model: bearing 1 


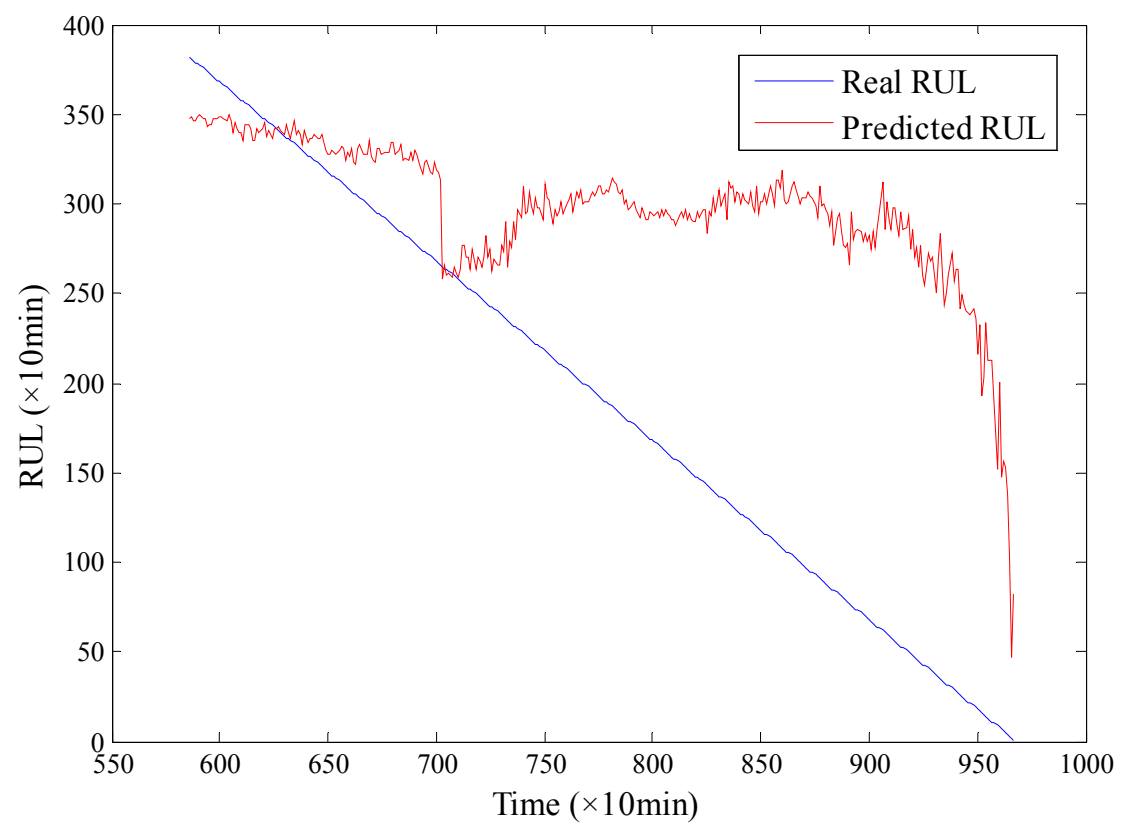

Fig.15. RUL prediction using traditional Paris crack growth model: bearing 1 


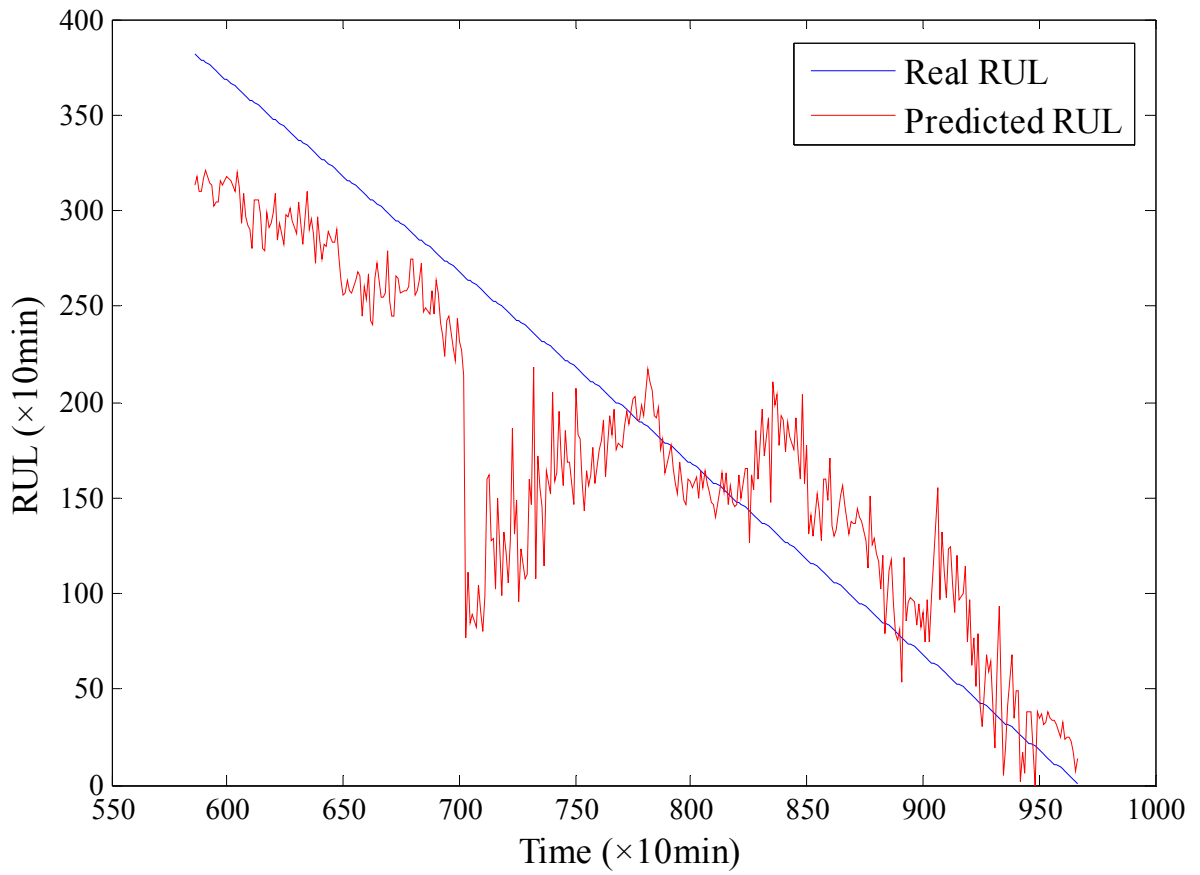

Fig. 16. RUL prediction using threshold-adjustment Paris crack growth model: bearing 1 


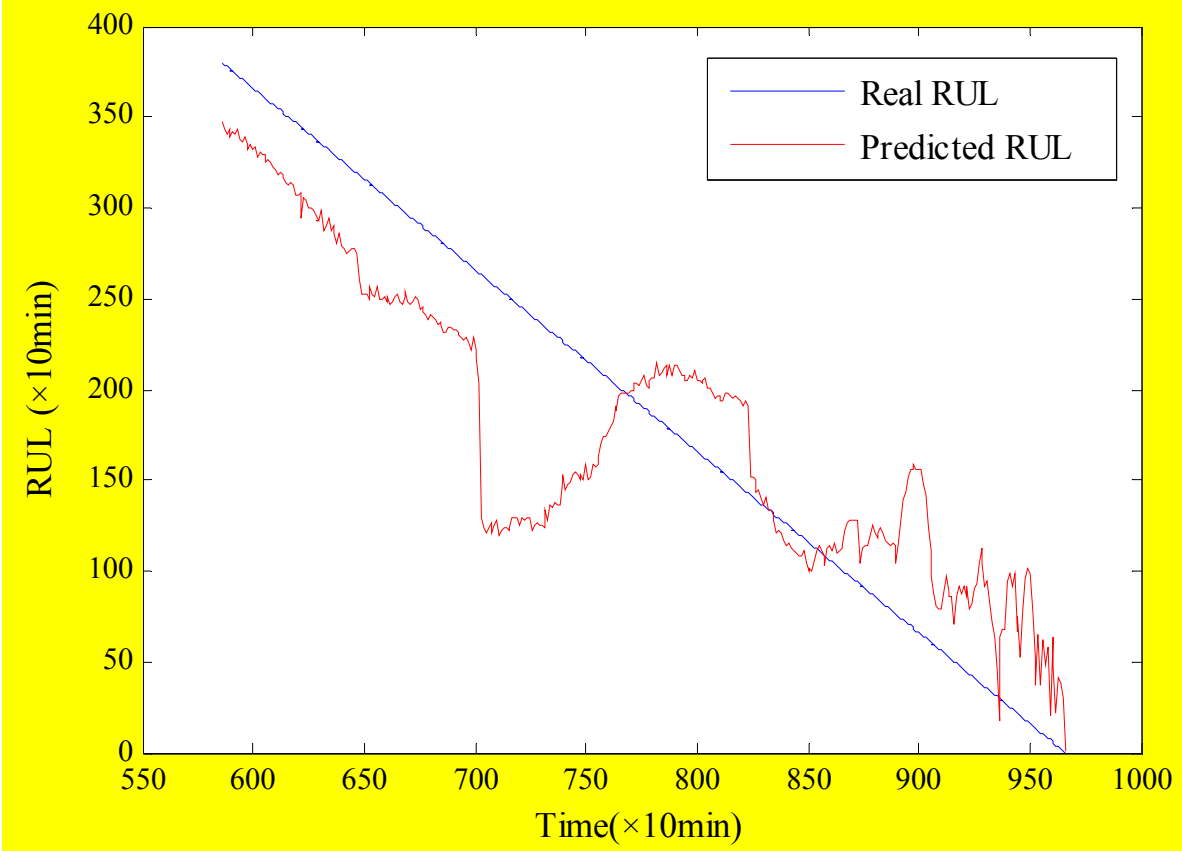

Fig. 17. RUL prediction using Paris crack growth model integrated with particle filter: bearing 1 


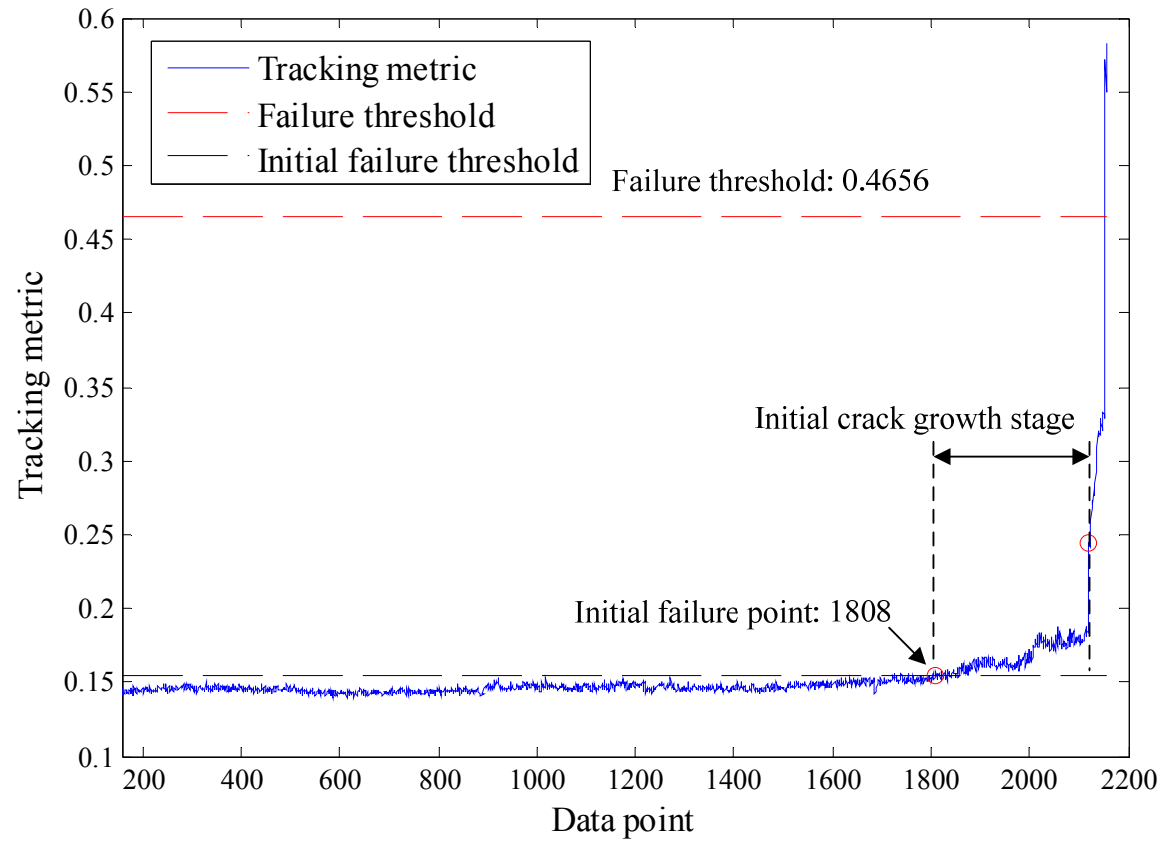

Fig.18. Degradation curve using enhanced PSW: bearing 3 


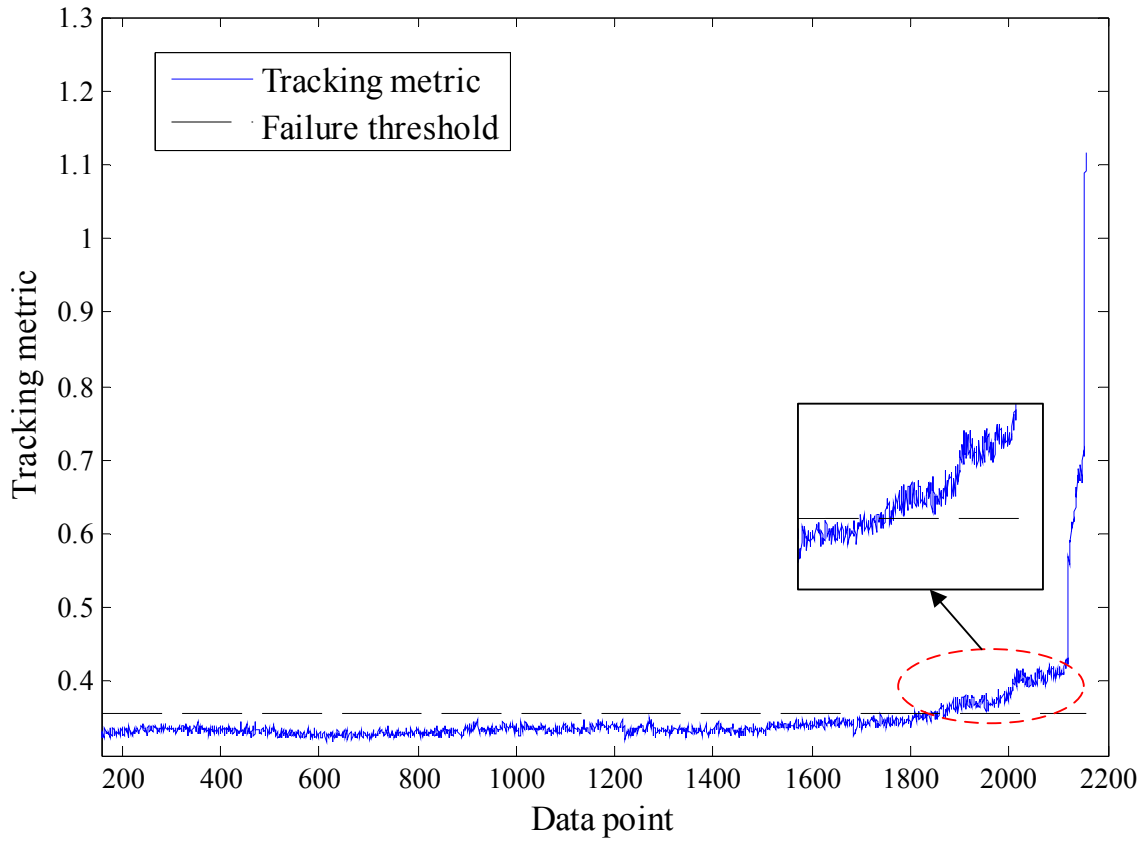

Fig. 19. Degradation curve using traditional PSW: bearing 3 


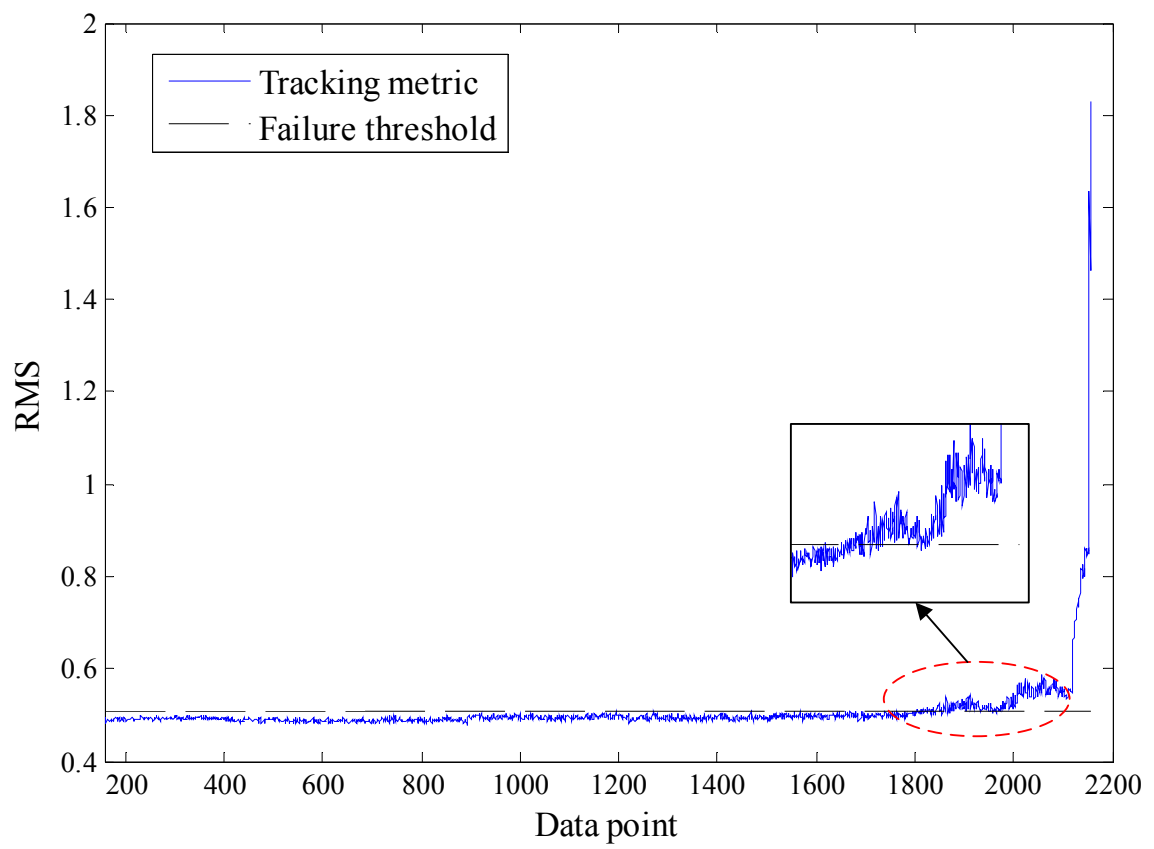

Fig.20. Degradation curve using RMS: bearing 3 


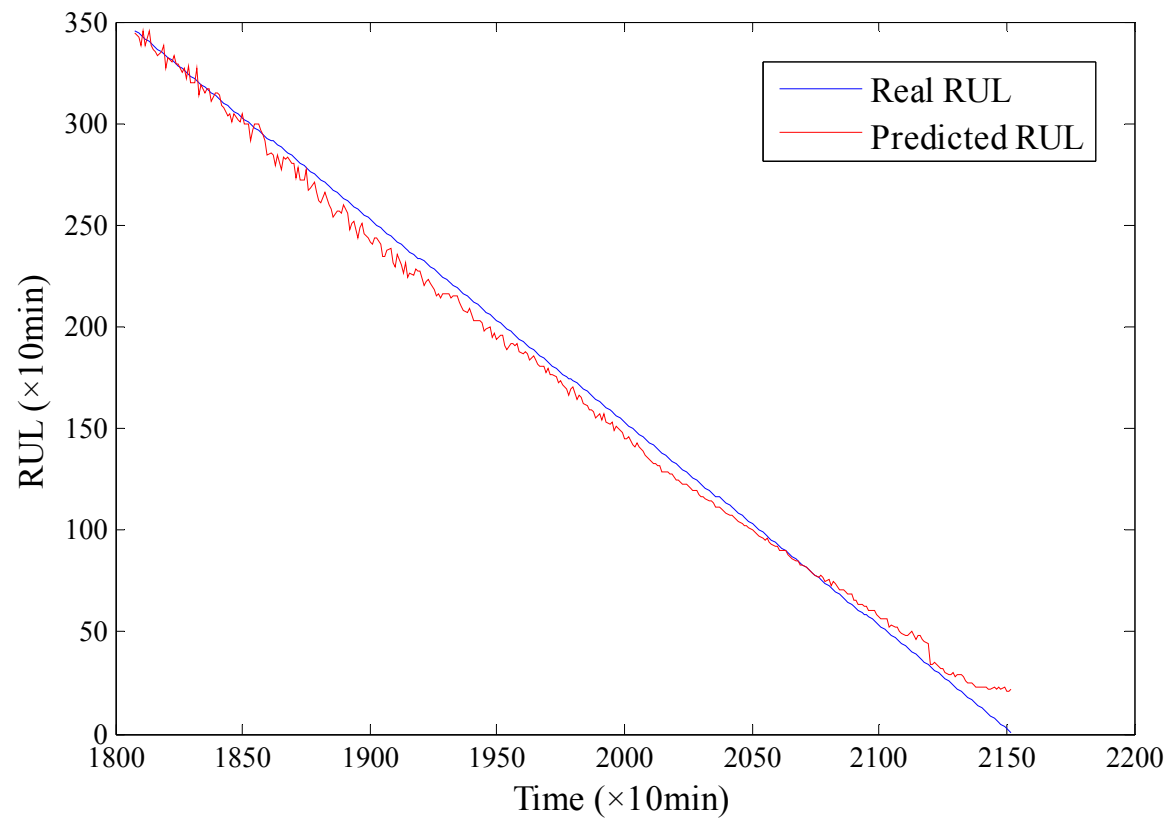

Fig. 21. RUL prediction using modified Paris crack growth model: bearing 3 


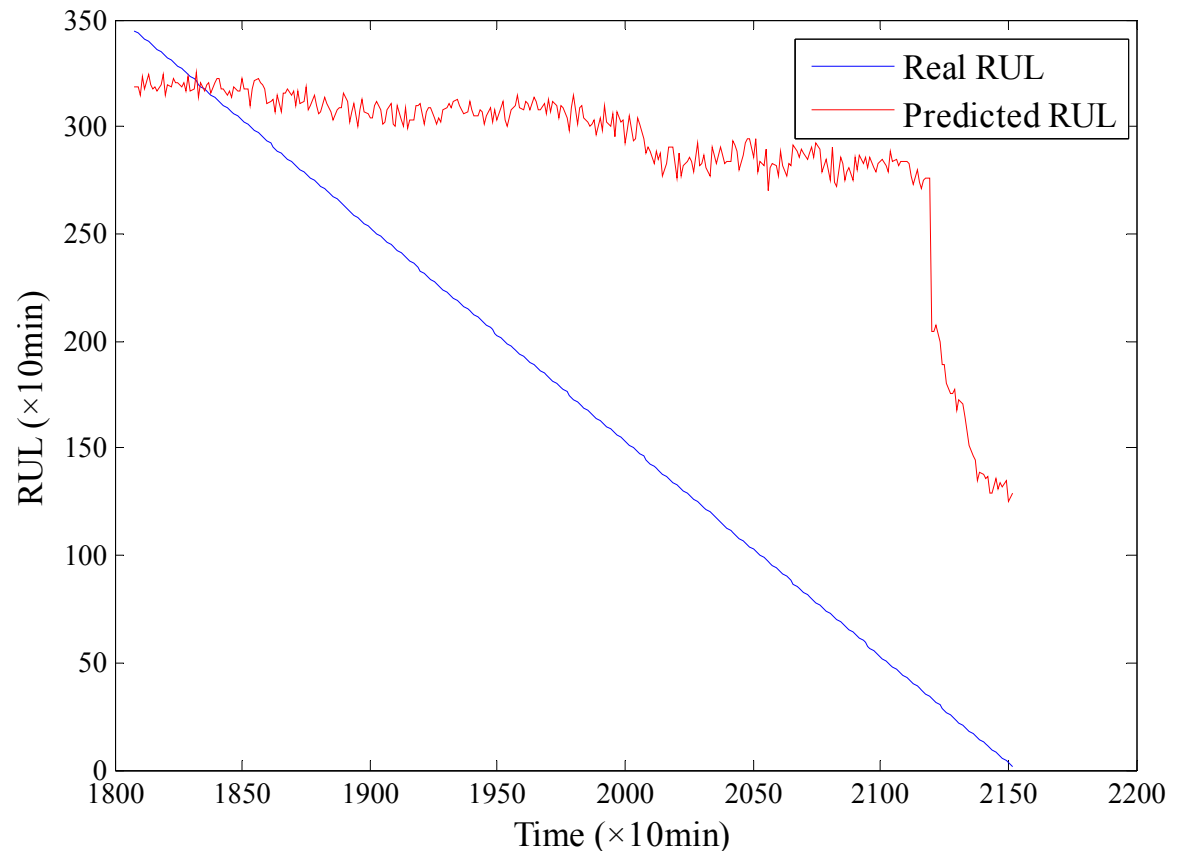

Fig.22. RUL prediction using traditional Paris crack growth model: bearing 3 


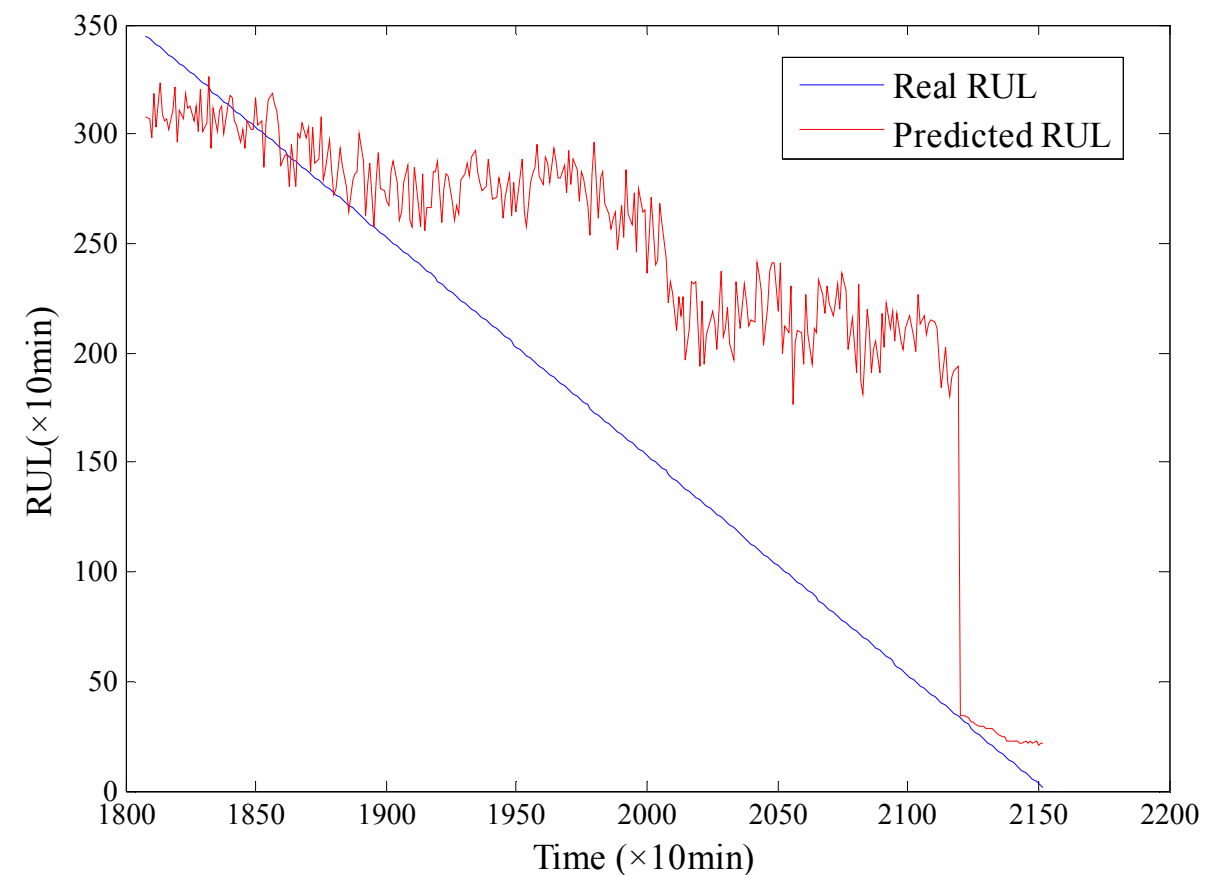

Fig. 23. RUL prediction using threshold-adjustment Paris crack growth model: bearing 3 


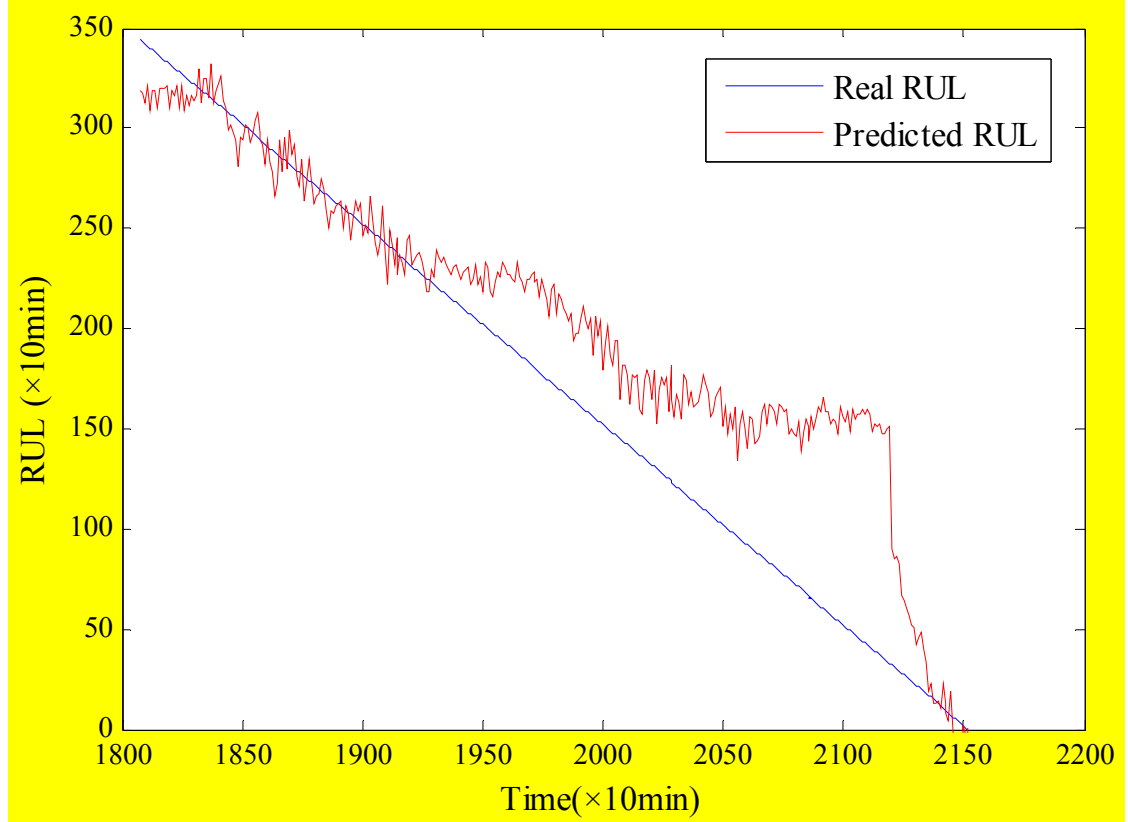

Fig. 24. RUL prediction using Paris crack growth model integrated with particle filter: bearing 3 